\title{
Delay-Dependent $H_{\infty}$ Control for Descriptor Markovian Jump Systems with Time-Varying Delay
}

\author{
Jinghao Li, ${ }^{1}$ Qingling Zhang, ${ }^{1}$ Ding Zhai, ${ }^{1}$ and Yi Zhang ${ }^{1,2}$ \\ ${ }^{1}$ Institute of Systems Science, Northeastern University, Shenyang 110819, China \\ ${ }^{2}$ School of Science, Shenyang University of Technology, Shenyang 110870, China \\ Correspondence should be addressed to Qingling Zhang; qlzhang@mail.neu.edu.cn
}

Received 11 August 2013; Accepted 20 September 2013

Academic Editor: Baolin Wang

Copyright (c) 2013 Jinghao Li et al. This is an open access article distributed under the Creative Commons Attribution License, which permits unrestricted use, distribution, and reproduction in any medium, provided the original work is properly cited.

\begin{abstract}
This paper is concerned with the delay-dependent $H_{\infty}$ control problem for continuous-time descriptor Markovian jump systems with time-varying delay. By constructing various Lyapunov-Krasovskii functionals for different subsystems, together with delay decomposition method, a new delay-dependent bounded real lemma (BRL) is derived, under which descriptor Markovian jump time-delay systems are regular, impulse-free, and stochastically stable and satisfy a prescribed $H_{\infty}$ performance level. Since the reciprocally convex combination approach is adopted to estimate the upper bound of the integral terms, the BRL obtained in this paper is less conservative than some existing ones. Based on the proposed BRL, a sufficient condition for the existence of state feedback controller is provided. Finally, three numerical examples are provided to demonstrate the validity of the proposed methods.
\end{abstract}

\section{Introduction}

Over the past decades, stability problems for time-delay systems have received considerable attention. Many methods have been presented to determine the stability. In the published results, two types of criteria have been widely investigated (see [1]), that is, delay-independent one and delay-dependent one. As we know, the delay-dependent criteria are less conservative than delay-independent ones, especially when the time delay is fairly small. Therefore, in order to reduce the conservatism of stability conditions, a great number of delay-dependent results have been reported, including, descriptor modeled transformation method (e.g., [2]), parameterized modeled transformation one (e.g., [3]), free-weighting matrix one (e.g., [4]), discretized Lyapunov functional one (e.g., [5]), delay decomposition one (e.g., [6]) and so on (e.g., [7, 8]). Among the aforementioned methods, since discretized Lyapunov functional method is complicated and it is difficult to apply this method to design controller, the delay decomposition method is less conservative than others except the discretized Lyapunov functional method.
Markovian jump systems are modeled by many stochastic systems, which arise abrupt random changes in their structure, such as component failures or repairs and unexpected environmental changes. A great deal of attention has been devoted to Markovian jump systems (see $[9,10]$ ). Recently, many efforts have been made to study the stability analysis and controller synthesis problems for Markovian jump systems with time-varying and time-invariant delay. Reference [11] divides the time-invariant delay into $m$ segments and uses the delay division method to obtain a less conservative criterion than [12]. In [13], stability and stabilization are discussed for Markovian jump systems with time-varying delay by selecting distinct Lyapunov matrices for different subsystems and introducing a triple-integral term. Reference [14] studies the $H_{\infty}$ analysis for Markovian jump stochastic systems with time-varying delay. By dividing the delay interval into two parts, two improved delay-dependent conditions have been presented to guarantee the stochastic stability.

Descriptor systems are also referred to as singular systems, semistate systems, which are a natural representation of dynamical systems and describe a much wider range of 
systems than standard state-space systems (see, e.g., [10, 15]). When descriptor systems are subject to time delay and stochastic disturbance in their structure, it is very natural to cast this model into descriptor Markovian jump time-delay systems. Very recently, a large number of researches have been published to study descriptor Markovian jump timedelay systems. By constructing more complicated LyapunovKrasovskii functionals, [16] studies the passivity and stochastic stability of descriptor Markovian jump systems with timevarying delay. Based on the delay decomposition method, [17] investigates the $H_{\infty}$ control problem for descriptor Markovian jump time-delay systems. To the best of our knowledge, the time-varying delay in most published results has not been tackled adequately, which leaves us much room to improve. All of these motivate the current study.

In this paper, we investigate $H_{\infty}$ control problem for continuous-time descriptor Markovian jump systems with time-varying delay. Motivated by delay decomposition method, through designing various Lyapunov-Krasovskii functionals for different subsystems, a delay-dependent BRL is established, which guarantees that the considered system is stochastically admissible and satisfies a prescribed $H_{\infty}$ performance level. The reciprocally convex combination method is used in the proof of the proposed results, which is one of the contributions to this paper. The other is the employed Lyapunov-Krasovskii functionals. Thanks to all of these, the obtained results are less conservative than some existing results.

The rest of this paper is organized as follows. Section 2 gives problem description and some essential lemmas. Section 3 focuses on $H_{\infty}$ control problem for continuoustime descriptor Markovian jump time-delay systems. A new delay-dependent BRL is proposed, and the design method for the state feedback controller, which guarantees that the resultant closed-loop system is stochastically admissible with a prescribed $H_{\infty}$ performance level, is provided. Section 4 provides numerical examples to illustrate the effectiveness of our methods, and Section 5 concludes the paper.

Notation. The notation used throughout this paper is quite standard. $\mathbb{R}^{n}$ stands for the $n$-dimensional Euclidean space, and $\mathbb{R}^{m \times n}$ represents the set of all $m \times n$ real matrices. The superscripts $T$ and -1 stand for matrix transposition and matrix inverse, respectively. $\mathscr{L}_{2}[0, \infty)$ represents the space of square integrable vector functions over the interval $[0, \infty)$. $(\Omega, \mathscr{F}, \mathscr{P})$ is the probability space, $\Omega$ is the sample space, $\mathscr{F}$ is the $\sigma$-algebra of subsets of the sample space, and $\mathscr{P}$ is the probability measure on $\mathscr{F} \cdot \mathscr{E}\{\cdot\}$ is the expectation operator with respect to some probability measure $\mathscr{P} . \mathbb{N}^{+}$represents the set of positive integers. The notation $P>0(P \geq 0)$ implies that $P$ is a real symmetric and positive definite (semipositive definite) matrix. For simplicity, $M_{i}$ is often adopted to denote $M(i)$. The asterisk * in matrix block implies that it can be induced by symmetric position. A block diagonal matrix with diagonal blocks $N_{1}, N_{2}, \ldots, N_{m}$ will be denoted by $\operatorname{diag}\left\{N_{1}, N_{2}, \ldots, N_{m}\right\}$. Matrices, if their dimensions are not explicitly stated, are assumed to be compatible for algebraic operations.

\section{Problem Formulation and Preliminaries}

Fix the probability space $(\Omega, \mathscr{F}, \mathscr{P})$ and consider the following continuous-time descriptor Markovian jump systems with time-varying delay:

$$
\begin{aligned}
E \dot{x}(t)= & A\left(r_{t}\right) x(t)+A_{d}\left(r_{t}\right) x(t-h(t)) \\
& +B\left(r_{t}\right) u(t)+B_{w}\left(r_{t}\right) w(t), \\
z(t)= & C\left(r_{t}\right) x(t)+C_{d}\left(r_{t}\right) x(t-h(t)) \\
& +D\left(r_{t}\right) u(t)+D_{w}\left(r_{t}\right) w(t), \\
x(\theta)= & \phi(\theta), \quad \forall \theta \in[-h, 0],
\end{aligned}
$$

where $x(t) \in \mathbb{R}^{n}$ is the state vector, $u(t) \in \mathbb{R}^{m}$ is the input vector, $z(t) \in \mathbb{R}^{q}$ is the controlled output vector, and $w(t) \in \mathbb{R}^{p}$ is the disturbance input vector which belongs to $\mathscr{L}_{2}[0, \infty) . E$ is a known real matrix which may be singular, and it is generally assumed that $\operatorname{rank}(E)=r \leq n$. The jumping mode $\left\{r_{t}, t \geq 0\right\}$, which takes value in a finite set $\ell=\{1,2, \ldots, N\}$, is the continuous-time Markov process with the following mode transition probabilities:

$$
\mathscr{P}\left(r_{t+\Delta}=j \mid r_{t}=i\right)= \begin{cases}\gamma_{i j} \Delta+o(\Delta), & \text { if } j \neq i \\ 1+\gamma_{i i} \Delta+o(\Delta), & \text { if } j=i,\end{cases}
$$

where $\Delta>0, \lim _{t \rightarrow 0}(o(\Delta) / \Delta)=0$, and $\gamma_{i j} \geq 0(i, j \in$ $\ell, j \neq i)$, satisfying $\gamma_{i i}=-\sum_{j \in \ell, j \neq i} \gamma_{i j}$, represent the switching rate from mode $i$ at time $t$ to mode $j$ at time $t+\Delta$ for all $i \in \ell . A\left(r_{t}\right), A_{d}\left(r_{t}\right), B\left(r_{t}\right), B_{w}\left(r_{t}\right), C\left(r_{t}\right), C_{d}\left(r_{t}\right), D\left(r_{t}\right)$, and $D_{w}\left(r_{t}\right)$ are known real constant matrices representing the deterministic systems for each $r_{t} \in \ell$. The considered timevarying delay $h(t)$ satisfies

$$
0 \leq h(t) \leq h, \quad \dot{h}(t) \leq h_{d},
$$

where $h$ and $h_{d}$ are known constants. Throughout this paper, for each $r_{t}=i \in \ell$, we use $M_{i}(t)$ and $N_{i}$ to denote matrices $M\left(r_{t}, t\right)$ and $N\left(r_{t}\right)$.

To facilitate exposition, the unforced descriptor Markovian jump time-delay systems are given as follows:

$$
\begin{gathered}
E \dot{x}(t)=A\left(r_{t}\right) x(t)+A_{d}\left(r_{t}\right) x(t-h(t))+B_{w}\left(r_{t}\right) w(t) \\
z(t)=C\left(r_{t}\right) x(t)+C_{d}\left(r_{t}\right) x(t-h(t))+D_{w}\left(r_{t}\right) w(t) \\
x(\theta)=\phi(\theta), \quad \forall \theta \in[-h, 0] .
\end{gathered}
$$

In the sequel, we recall some indispensable definitions.

Definition 1 (see [10]). When the disturbance signal $w(t) \equiv 0$, for any given scalars $h>0$ and $h_{d}$, consider the following statements.

(1) The continuous-time descriptor Markovian jump time-delay systems (4) are said to be regular and impulse-free for any time-varying delay satisfying (3) if the pairs $\left(E, A_{i}\right)$ are regular and impulse-free for each $r_{t} \in \ell$. 
(2) The continuous-time descriptor Markovian jump time-delay systems (4) are said to be stochastically stable if there exists a scalar $M\left(\phi(\cdot), r_{0}\right)>0$ such that

$$
\begin{gathered}
\lim _{t \rightarrow \infty} \mathscr{E}\left\{\int_{0}^{t} x^{T}\left(s, x_{t}, r_{0}\right) x\left(s, x_{t}, r_{0}\right) d s \mid x_{t}=x(s)=\phi(s),\right. \\
\left.s \in[-h, 0], r_{0} \in \ell\right\} \leq M\left(\phi(\cdot), r_{0}\right)
\end{gathered}
$$

where $x\left(t, x_{t}, r_{0}\right)$ denotes the solution to systems (4) at time $t$ under the given initial conditions.

(3) The continuous-time descriptor Markovian jump time-delay systems (4) are said to be stochastically admissible if they are regular, impulse-free, and stochastically stable.

Definition 2 (see [17]). For a given scalar $\gamma>0$, the descriptor Markovian jump time-delay systems (4) are said to be stochastically admissible with $H_{\infty}$ performance level $\gamma$; if the system with $w(t) \equiv 0$ is stochastically admissible and under zero initial condition, it satisfies

$$
\mathscr{E}\left\{\int_{0}^{\infty} z^{T}(t) z(t) d t\right\} \leq \gamma^{2} \int_{0}^{\infty} w^{T}(t) w(t) d t
$$

for any nonzero $w(t) \in \mathscr{L}[0, \infty)$.

In this paper, the purpose is to design a mode-dependent state feedback controller for the continuous-time descriptor Markovian jump time-delay systems (1) with a prescribed scalar $\gamma>0$ such that the resultant closed-loop systems are stochastically admissible with $H_{\infty}$ performance level $\gamma$. Specifically, the desired state feedback controller can be expressed as

$$
u(t)=K\left(r_{t}\right) x(t)+K_{d}\left(r_{t}\right) x(t-h(t)) .
$$

To this end, some essential lemmas are introduced to expedite the following discussion.

Lemma 3 (see [18]). For any constant matrix $W \in \mathbb{R}^{n \times n}, W>$ 0 , scalar $\gamma>0$, and vector function $x:[-\gamma, 0] \rightarrow \mathbb{R}^{n}$ such that the following integration is well defined, then

$$
-\gamma \int_{t-\gamma}^{t} x^{T}(s) W x(s) d s \leq-\int_{t-\gamma}^{t} x^{T}(s) d s W \int_{t-\gamma}^{t} x(s) d s .
$$

Lemma 4 (see $[8]$ ). Let $f_{1}, f_{2}, \ldots, f_{N}: \mathbb{R}^{m} \mapsto \mathbb{R}$ have positive values in an open subset $\mathbb{D}$ of $\mathbb{R}^{m}$. Then the reciprocally convex combination of $f_{i}$ over $\mathbb{D}$ satisfies

$$
\min _{\left\{\alpha_{i} \mid \alpha_{i}>0, \sum_{i} \alpha_{i}=1\right\}} \sum_{i} \frac{1}{\alpha_{i}} f_{i}(t)=\sum_{i} f_{i}(t)+\max _{g_{i, j}(t)} \sum_{i \neq j} g_{i, j}(t),
$$

subject to

$$
\left\{g_{i, j}: \mathbb{R}^{m} \longmapsto \mathbb{R}, g_{j, i}(t)=g_{i, j}(t),\left[\begin{array}{cc}
f_{i}(t) & g_{i, j}(t) \\
g_{i, j}(t) & f_{i}(t)
\end{array}\right] \geq 0\right\} .
$$

Based on Lemma 4, we present the following lemma, which can be regarded as an alternative form of the reciprocally convex combination.

Lemma 5. For any constant matrix $W \in \mathbb{R}^{n \times n}, W \geq 0$, and a variable $\beta$ satisfying $a \leq \beta \leq b$, if there exists a matrix $\widehat{W}$ satisfying $\left[\begin{array}{cc}W & \widehat{W} \\ \widehat{W}^{T} & W\end{array}\right] \geq 0$, then

$$
\begin{gathered}
-\frac{1}{\beta-a} e_{1}(t)^{T} W e_{1}(t)-\frac{1}{b-\beta} e_{2}^{T}(t) W e_{2}(t) \\
\leq-\frac{1}{b-a}\left[\begin{array}{l}
e_{1}(t) \\
e_{2}(t)
\end{array}\right]^{T}\left[\begin{array}{cc}
W & \widehat{W} \\
\widehat{W}^{T} & W
\end{array}\right]\left[\begin{array}{l}
e_{1}(t) \\
e_{2}(t)
\end{array}\right],
\end{gathered}
$$

where $e_{1}(t)$ and $e_{2}(t)$ are vector functions with compatible dimensions.

Proof. Taking

$$
\begin{gathered}
f_{1}(t)=e_{1}^{T}(t) W e_{1}(t), \\
f_{2}(t)=e_{2}^{T}(t) W e_{2}(t), \\
g_{1,2}(t)=e_{1}^{T}(t) \widehat{W} e_{2}(t), \\
g_{2,1}(t)=e_{2}^{T}(t) \widehat{W}^{T} e_{1}(t), \\
\alpha_{1}=\frac{\beta-a}{b-a}, \quad \alpha_{2}=\frac{b-\beta}{b-a},
\end{gathered}
$$

it can be easily verified that the above choice satisfies constraint (10) in Lemma 4. Note that with the equality (9), we have

$$
\begin{gathered}
\min _{\left\{\alpha_{i} \mid \alpha_{i}>0, \sum_{i} \alpha_{i}=1\right\}} \sum_{i} \frac{1}{\alpha_{i}} f_{i}(t) \\
\leq \frac{b-a}{\beta-a} e_{1}^{T}(t) W e_{1}(t)+\frac{b-a}{b-\beta} e_{2}^{T}(t) W e_{2}(t), \\
\sum_{i} f_{i}(t)+\max _{g_{i, j}(t)} \sum_{i \neq j} g_{i, j}(t) \geq\left[\begin{array}{l}
e_{1}(t) \\
e_{2}(t)
\end{array}\right]^{T}\left[\begin{array}{cc}
W & \widehat{W} \\
\widehat{W}^{T} & W
\end{array}\right]\left[\begin{array}{l}
e_{1}(t) \\
e_{2}(t)
\end{array}\right] .
\end{gathered}
$$

By Lemma 4, (13) and (14) yield that

$$
\begin{gathered}
-\frac{1}{\beta-a} e_{1}^{T}(t) W e_{1}(t)-\frac{1}{b-\beta} e_{2}^{T}(t) W e_{2}(t) \\
\leq-\frac{1}{b-a}\left[\begin{array}{l}
e_{1}(t) \\
e_{2}(t)
\end{array}\right]^{T}\left[\begin{array}{cc}
W & \widehat{W} \\
\widehat{W}^{T} & W
\end{array}\right]\left[\begin{array}{l}
e_{1}(t) \\
e_{2}(t)
\end{array}\right] .
\end{gathered}
$$

This completes the proof.

Lemma 6 (see [10]). Given any real matrices $S_{1}, S_{2}$, and $S_{3}$ with $S_{1}^{T}=S_{1}$ and $S_{3}>0$, then one has

$$
S_{1}+S_{2} S_{3}^{-1} S_{2}^{T}<0
$$

if and only if

$$
\left[\begin{array}{cc}
S_{1} & S_{2} \\
S_{2}^{T} & -S_{3}
\end{array}\right]<0
$$


or equivalently

$$
\left[\begin{array}{cc}
-S_{3} & S_{2}^{T} \\
S_{2} & S_{1}
\end{array}\right]<0 .
$$

Lemma 7 (see [10]). Given matrices $\Phi=\Phi^{T}<0, D$ and $E$ with appropriate dimensions

$$
\Phi+D F(t) E+E^{T} F(t)^{T} D^{T}<0
$$

for all $F(t)$ satisfying $F^{T}(t) F(t) \leq I$ if and only if there exists scalar $\epsilon>0$ such that

$$
\Phi+\varepsilon D D^{T}+\varepsilon^{-1} E^{T} E<0
$$

Lemma 8 (see [19]). Let $P$ be symmetric such that $E_{R}^{T} P E_{R}>0$, and let $\Phi$ be nonsingular. Then, $P E+U^{T} \Phi V^{T}$ is nonsingular and its inverse is expressed as

$$
\left(P E+U^{T} \Phi V^{T}\right)^{-1}=\bar{P} E^{T}+V \bar{\Phi} U
$$

where $E_{L}$ and $E_{R}$ are full rank with $E=E_{L} E_{R}^{T}$ and $U^{T}$ and $V$ are of full column rank and composed of bases of null space of $E^{T}$ and $E$, respectively. $\bar{P}$ is symmetric and $\bar{\Phi}$ is nonsingular such that $E_{R}^{T} \bar{P} E_{R}=\left(E_{L}^{T} P E_{L}\right)^{-1}, \bar{\Phi}=\left(V^{T} V\right)^{-1} \Phi^{-1}\left(U U^{T}\right)^{-1}$.

\section{Main Results}

In this section, the delay-dependent $H_{\infty}$ control problem will be studied for the descriptor Markovian jump time-delay system (1) by virtue of the delay decomposition method. To this end, by dividing the delay interval $(0, h]$ into two disjoint subintervals $(0, \alpha h]$ and $(\alpha h, h],(0<\alpha<1)$, the following delay-dependent bounded real lemma (BRL) for the system (4) is firstly proposed, which will play an indispensable role in the derivation of our main results.

Theorem 9. For given scalars $\gamma>0, h>0$, and $0 \leq h_{d}<$ 1, the descriptor Markovian jump time-delay system (4) is stochastically admissible with $H_{\infty}$ performance level $\gamma$ for any $h(t)$ satisfying (3), if there exist a set of positive definite matrices $P_{i}, Q_{1 i}, Q_{2 i}, Q_{3 i}, R_{2 i}, R_{3 i}, U_{1}, U_{2}, W$, and matrices $\Phi_{i}, S_{i}, T_{i}$, $\forall i \in \ell$, satisfying the following LMIs for each $i \in \ell$ :

$$
\left.\begin{array}{cccccccccc}
\Theta_{i 11} & \Theta_{i 12} & \Theta_{i 13} & 0 & \Theta_{i 15} & A_{i}^{T} R_{2 i} & A_{i}^{T} R_{3 i} & \frac{1}{2} h^{2} A_{i}^{T} W & C_{i}^{T} \\
* & \Theta_{i 22} & \Theta_{i 23} & 0 & 0 & A_{d i}^{T} R_{2 i} & A_{d i}^{T} R_{3 i} & \frac{1}{2} h^{2} A_{d i}^{T} W & C_{d i}^{T} \\
* & * & \Theta_{i 33} & \Theta_{i 34} & 0 & 0 & 0 & 0 & 0 \\
* & * & * & \Theta_{i 44} & 0 & 0 & 0 & 0 & 0 \\
* & * & * & * & -\gamma^{2} I & B_{w i}^{T} R_{2 i} & B_{w i}^{T} R_{3 i} & \frac{1}{2} h^{2} B_{w i}^{T} W & D_{w i}^{T} \\
* & * & * & * & * & -\alpha_{1} R_{2 i} & 0 & 0 & 0 \\
* & * & * & * & * & * & -\alpha_{2} R_{3 i} & 0 & 0 \\
* & * & * & * & * & * & * & -\frac{1}{2} h^{2} W & 0 \\
* & * & * & * & * & * & * & * & -I
\end{array}\right]<0, \quad h(t) \in(0, \alpha h]
$$




$$
Z_{1 i}=\left[\begin{array}{cc}
R_{2 i} & S_{i} \\
S_{i}^{T} & R_{2 i}
\end{array}\right]>0, \quad Z_{2 i}=\left[\begin{array}{cc}
R_{3 i} & T_{i} \\
T_{i}^{T} & R_{3 i}
\end{array}\right]>0,
$$

where $\alpha_{1}=1 / \alpha h, \alpha_{2}=1 /(h-\alpha h)$, and

$$
\begin{aligned}
\Theta_{i 11}= & A_{i}^{T}\left(P_{i} E+U^{T} \Phi_{i} V^{T}\right)+\left(P_{i} E+U^{T} \Phi_{i} V^{T}\right)^{T} A_{i} \\
& +\sum_{j=1}^{N} \gamma_{i j} E^{T} P_{j} E+Q_{1 i}+Q_{2 i} \\
& +\alpha h U_{1}+(1-\alpha) h U_{2}-\alpha_{1} E^{T} R_{2 i} E \\
\Theta_{i 12}= & \left(P_{i} E+U^{T} \Phi_{i} V^{T}\right)^{T} A_{d i}+\alpha_{1} E^{T} R_{2 i} E-\alpha_{1} E^{T} S_{i} E \\
\Theta_{i 13}= & \alpha_{1} E^{T} S_{i} E, \\
\Theta_{i 15}= & \left(P_{i} E+U^{T} \Phi_{i} V^{T}\right)^{T} B_{w i}, \\
\Theta_{i 22}= & -\left(1-h_{d}\right) Q_{1 i}-2 \alpha_{1} E^{T} R_{2 i} E+\alpha_{1} E^{T} S_{i} E+\alpha_{1} E^{T} S_{i}^{T} E, \\
\Theta_{i 23}= & \alpha_{1} E^{T} R_{2 i} E-\alpha_{1} E^{T} S_{i} E \\
\Theta_{i 33}= & Q_{3 i}-Q_{2 i}-\alpha_{1} E^{T} R_{2 i} E-\alpha_{2} E^{T} R_{3 i} E \\
\Theta_{i 34}= & \alpha_{2} E^{T} R_{3 i} E, \\
\Theta_{i 44}= & -Q_{3 i}-\alpha_{2} E^{T} R_{3 i} E \\
\widehat{\Theta}_{i 12}= & \left(P_{i} E+U^{T} \Phi_{i} V^{T}\right)^{T} A_{d i}, \\
\widehat{\Theta}_{i 13}= & \alpha_{1} E^{T} R_{2 i} E, \\
\widehat{\Theta}_{i 22}= & -\left(1-h_{d}\right) Q_{1 i}-2 \alpha_{2} E^{T} R_{3 i} E+\alpha_{2} E^{T} T_{i} E+\alpha_{2} E^{T} T_{i}^{T} E, \\
\widehat{\Theta}_{i 23}= & \alpha_{2} E^{T} R_{3 i} E-\alpha_{2} E^{T} T_{i}^{T} E, \\
\widehat{\Theta}_{i 24}= & \alpha_{2} E^{T} R_{3 i} E-\alpha_{2} E^{T} T_{i} E \\
\widehat{\Theta}_{i 34}= & \alpha_{2} E^{T} T_{i} E \\
& \\
& \\
&
\end{aligned}
$$

Proof. Assume that there exist matrices such that LMIs (22)(27) hold. With these conditions in hand, we firstly show the regularity and absence of impulse of the system (4). To this end, it is from [10] that there exist a couple of nonsingular matrices $M$ and $N$ such that for each $i \in \ell$

$$
\begin{gathered}
\operatorname{MEN}=\left[\begin{array}{cc}
I_{r} & 0 \\
0 & 0
\end{array}\right], \quad M A_{i} N=\left[\begin{array}{ll}
A_{i 1} & A_{i 2} \\
A_{i 3} & A_{i 4}
\end{array}\right], \\
M A_{d i} N=\left[\begin{array}{ll}
A_{d i 1} & A_{d i 2} \\
A_{d i 3} & A_{d i 4}
\end{array}\right] .
\end{gathered}
$$

Decomposing the associated matrices with a compatible dimension, we have

$$
\begin{aligned}
M^{-T} P_{i} M^{-1}= & {\left[\begin{array}{ll}
P_{i 1} & P_{i 2} \\
P_{i 3} & P_{i 4}
\end{array}\right], \quad M^{-T} U^{T}=\left[\begin{array}{l}
0 \\
I
\end{array}\right] Z_{1}, } \\
& V^{T} N=Z_{2}\left[\begin{array}{ll}
0 & I
\end{array}\right]
\end{aligned}
$$

where $Z_{1} \in \mathbb{R}^{(n-r) \times(n-r)}$ and $Z_{2} \in \mathbb{R}^{(n-r) \times(n-r)}$ are arbitrarily nonsingular matrices.
From the $(1,1)$ block in the left-hand matrices of $(22)$ or (23), and observing that $Q_{1 i}, Q_{2 i}, U_{1}$, and $U_{2}$ are positive definite, it can be easily seen that

$$
\begin{aligned}
A_{i}^{T}( & \left.P_{i} E+U^{T} \Phi_{i} V^{T}\right)+\left(P_{i} E+U^{T} \Phi_{i} V^{T}\right)^{T} A_{i} \\
& +E^{T} f\left(P_{j}, R_{2 i}\right) E<0,
\end{aligned}
$$

where $f\left(P_{j}, R_{2 i}\right)$ is the function of $P_{j}$ and $R_{2 i}$ and makes no difference to the subsequent proof.

Pre- and postmultiplying (31) by $N^{T}$ and $N$, respectively, together with (29) and (30), we get

$$
\left[\begin{array}{cc}
* & * \\
* & A_{i 4}^{T} Z_{1} \Phi_{i} Z_{2}+Z_{2}^{T} \Phi_{i}^{T} Z_{1}^{T} A_{i 4}
\end{array}\right]<0
$$

According to [10], we can know that the pairs $\left(E, A_{i}\right)$ are regular and impulse-free for each $i \in \ell$. Therefore, by Definition 1, the system (4) is regular and impulse-free.

Next, we will show the stochastic stability of the system (4). To this end, we define a new process $\left\{\left(x_{t}, r_{t}\right), t \geq 0\right\}$ by $\left\{x_{t}(s)=x(t+s),-2 h \leq s \leq 0\right\}$; then from [12], we can know $\left\{\left(x_{t}, r_{t}\right), t \geq h\right\}$ is a Markov process with initial state $\left(\phi(\cdot), r_{0}\right)$. Now, construct the stochastic Lyapunov functional candidate to be

$$
V\left(x_{t}, r_{t}, t\right)=V_{1}\left(x_{t}, r_{t}, t\right)+V_{2}\left(x_{t}, r_{t}, t\right)+V_{3}\left(x_{t}, r_{t}, t\right),
$$

where

$$
\begin{aligned}
& V_{1}\left(x_{t}, r_{t}, t\right)= x^{T}(t) E^{T} P\left(r_{t}\right) E x(t), \\
& V_{2}\left(x_{t}, t\right)= \int_{t-h(t)}^{t} x^{T}(s) Q_{1}\left(r_{t}\right) x(s) d s \\
&+\int_{t-\alpha h}^{t} x^{T}(s) Q_{2}\left(r_{t}\right) x(s) d s \\
&+\int_{t-h}^{t-\alpha h} x^{T}(s) Q_{3}\left(r_{t}\right) x(s) d s \\
&+\int_{-\alpha h}^{0} \int_{t+\theta}^{t} x^{T}(s) U_{1} x(s) d s d \theta \\
&+\int_{-h}^{-\alpha h} \int_{t+\theta}^{t} x^{T}(s) U_{2} x(s) d s d \theta, \\
&+\int_{-h}^{0} \int_{\beta}^{0} \int_{t+\theta}^{t} \dot{x}^{T}(s) E^{T} W E \dot{x}(s) d s d \theta d \beta . \\
&+\int_{-h}^{t} \dot{x}_{t+\theta}^{-\alpha h}(s) E_{t+\theta}^{t} \dot{x}^{T}(s) E_{2}\left(r_{t}\right) E \dot{x}(s) d s d \theta \\
& \\
&\left.V_{3}, t\right)=\left(r_{t}\right) E \dot{x}(s) d s d \theta
\end{aligned}
$$


Let $\mathscr{L}$ be the weak infinitesimal generator of the random process $\left\{x_{t}, t\right\}$ acting on $V\left(x_{t}, r_{t}, t\right)$ [12]. Then for each $r_{t}=i \in$ $\ell$, it can be shown that

$$
\begin{aligned}
& \mathscr{L} V_{1}\left(x_{t}, i, t\right) \\
&=\dot{x}^{T}(t) E^{T} P_{i} E x(t)+x^{T}(t) E^{T} P_{i} E \dot{x}(t) \\
&+x^{T}(t)\left(\sum_{j=1}^{N} \gamma_{i j} E^{T} P_{j} E\right) x(t) \\
&= \dot{x}^{T}(t) E^{T}\left(P_{i} E+U^{T} \Phi_{i} V^{T}\right) x(t) \\
&+x^{T}(t)\left(P_{i} E+U^{T} \Phi_{i} V^{T}\right)^{T} E \dot{x}(t) \\
&+x^{T}(t)\left(\sum_{j=1}^{N} \gamma_{i j} E^{T} P_{j} E\right) x(t) \\
&= x^{T}(t) A_{i}^{T}\left(P_{i} E+U^{T} \Phi_{i} V^{T}\right) x(t) \\
&+x^{T}(t-h(t)) A_{d i}^{T}\left(P_{i} E+U^{T} \Phi_{i} V^{T}\right) x(t) \\
&+x^{T}(t)\left(P_{i} E+U^{T} \Phi_{i} V^{T}\right)^{T} A_{i} x(t) \\
&+x^{T}(t)\left(P_{i} E+U^{T} \Phi_{i} V^{T}\right)^{T} A_{d i} x(t-h(t)) \\
&+x^{T}(t)\left(\sum_{j=1}^{N} \gamma_{i j} E^{T} P_{j} E\right) x(t) \\
&+x^{T}(t)\left(A_{i}^{T}\left(P_{i} E+U^{T} \Phi_{i} V^{T}\right)+\left(P_{i} E+U^{T} \Phi_{i} V^{T}\right)^{T} A_{i}\right. \\
&+x^{T}(t-h(t)) A_{d i}^{T}\left(P_{i} E+U^{T} \Phi_{i} V^{T}\right) x(t) \\
&\left.+\sum_{j=1}^{N} \gamma_{i j} E^{T} P_{j} E\right) x(t)\left(P_{i} E+U^{T} \Phi_{i} V^{T}\right)^{T} A_{d i} x(t-h(t)),
\end{aligned}
$$

$$
\begin{aligned}
\mathscr{L} V_{2} & \left(x_{t}, i, t\right) \\
= & x^{T}(t)\left(Q_{1 i}+Q_{2 i}+\alpha h U_{1}+(1-\alpha) h U_{2}\right) x(t) \\
& -(1-\dot{h}(t)) x^{T}(t-h(t)) Q_{1 i} x(t-h(t)) \\
& +x^{T}(t-\alpha h)\left(Q_{3 i}-Q_{2 i}\right) x(t-\alpha h) \\
& -x^{T}(t-h) Q_{3 i} x(t-h) \\
& +\int_{t-h(t)}^{t} x^{T}(s)\left(\sum_{j=1}^{N} \gamma_{i j} Q_{1 j}\right) x(s) d s \\
& +\int_{t-\alpha h}^{t} x^{T}(s)\left(\sum_{j=1}^{N} \gamma_{i j} Q_{2 j}\right) x(s) d s \\
& +\int_{t-h}^{t-\alpha h} x^{T}(s)\left(\sum_{j=1}^{N} \gamma_{i j} Q_{3 j}\right) x(s) d s \\
& -\int_{t-\alpha h}^{t} x^{T}(s) U_{1} x(s) d s \\
& -\int_{t-h}^{t-\alpha h} x^{T}(s) U_{2} x(s) d s
\end{aligned}
$$

$\leq x^{T}(t)\left(Q_{1 i}+Q_{2 i}+\alpha h U_{1}+(1-\alpha) h U_{2}\right) x(t)$

$-\left(1-h_{d}\right) x^{T}(t-h(t)) Q_{1 i} x(t-h(t))$

$+x^{T}(t-\alpha h)\left(Q_{3 i}-Q_{2 i}\right) x(t-\alpha h)$

$-x^{T}(t-h) Q_{3 i} x(t-h)$

$+\int_{t-h(t)}^{t} x^{T}(s)\left(\sum_{j=1}^{N} \gamma_{i j} Q_{1 j}\right) x(s) d s$

$+\int_{t-\alpha h}^{t} x^{T}(s)\left(\sum_{j=1}^{N} \gamma_{i j} Q_{2 j}\right) x(s) d s$

$+\int_{t-h}^{t-\alpha h} x^{T}(s)\left(\sum_{j=1}^{N} \gamma_{i j} Q_{3 j}\right) x(s) d s$

$-\int_{t-\alpha h}^{t} x^{T}(s) U_{1} x(s) d s$

$-\int_{t-h}^{t-\alpha h} x^{T}(s) U_{2} x(s) d s$,

$\mathscr{L} V_{3}\left(x_{t}, i, t\right)$

$=\dot{x}^{T}(t) E^{T}\left(\alpha h R_{2 i}+(1-\alpha) h R_{3 i}+\frac{1}{2} h^{2} W\right) E \dot{x}(t)$

$-\int_{t-\alpha h}^{t} \dot{x}^{T}(s) E^{T} R_{2 i} E \dot{x}(s) d s$

$-\int_{t-h}^{t-\alpha h} \dot{x}^{T}(s) E^{T} R_{3 i} E \dot{x}(s) d s$

$+\int_{-\alpha h}^{0} \int_{t+\theta}^{t} \dot{x}^{T}(s)\left(\sum_{j=1}^{N} \gamma_{i j} E^{T} R_{2 j} E\right) \dot{x}(s) d s d \theta$

$-\int_{-\alpha h}^{0} \int_{t+\theta}^{t} \dot{x}^{T}(s) E^{T} W E \dot{x}(s) d s d \theta$

$+\int_{-h}^{-\alpha h} \int_{t+\theta}^{t} \dot{x}^{T}(s)\left(\sum_{j=1}^{N} \gamma_{i j} E^{T} R_{3 j} E\right) \dot{x}(s) d s d \theta$

$-\int_{-h}^{-\alpha h} \int_{t+\theta}^{t} \dot{x}^{T}(s) E^{T} W E \dot{x}(s) d s d \theta$

$=\dot{x}^{T}(t) E^{T}\left(\alpha h R_{2 i}+(1-\alpha) h R_{3 i}+\frac{1}{2} h^{2} W\right) E \dot{x}(t)$

$-\int_{t-\alpha h}^{t} \dot{x}^{T}(s) E^{T} R_{2 i} E \dot{x}(s) d s$

$+\int_{-\alpha h}^{0} \int_{t+\theta}^{t} \dot{x}^{T}(s) E^{T}\left(\sum_{j=1}^{N} \gamma_{i j} R_{2 j}-W\right) E \dot{x}(s) d s d \theta$

$-\int_{t-h}^{t-\alpha h} \dot{x}^{T}(s) E^{T} R_{3 i} E \dot{x}(s) d s$

$+\int_{-h}^{-\alpha h} \int_{t+\theta}^{t} \dot{x}^{T}(s) E^{T}\left(\sum_{j=1}^{N} \gamma_{i j} R_{3 j}-W\right) E \dot{x}(s) d s d \theta$.

Next, we will estimate the upper bound of the integral terms in $\mathscr{L} V_{2}\left(x_{t}, i, t\right)$ and $\mathscr{L} V_{3}\left(x_{t}, i, t\right)$. 
Case $1(0<h(t) \leq \alpha h)$. The upper bound can be evaluated for the last five integral terms in $\mathscr{L} V_{2}\left(x_{t}, i, t\right)$, which can be shown as

$$
\begin{aligned}
\int_{t-h(t)}^{t} & x^{T}(s)\left(\sum_{j=1}^{N} \gamma_{i j} Q_{1 j}\right) x(s) d s \\
& +\int_{t-\alpha h}^{t} x^{T}(s)\left(\sum_{j=1}^{N} \gamma_{i j} Q_{2 j}\right) x(s) d s \\
& +\int_{t-h}^{t-\alpha h} x^{T}(s)\left(\sum_{j=1}^{N} \gamma_{i j} Q_{3 j}\right) x(s) d s \\
& -\int_{t-\alpha h}^{t} x^{T}(s) U_{1} x(s) d s-\int_{t-h}^{t-\alpha h} x^{T}(s) U_{2} x(s) d s \\
= & \int_{t-h(t)}^{t} x^{T}(s)\left(\sum_{j=1}^{N} \gamma_{i j} Q_{1 j}+\sum_{j=1}^{N} \gamma_{i j} Q_{2 j}-U_{1}\right) x(s) d s \\
& +\int_{t-\alpha h}^{t-h(t)} x^{T}(s)\left(\sum_{j=1}^{N} \gamma_{i j} Q_{2 j}-U_{1}\right) x(s) d s \\
& +\int_{t-h}^{t-\alpha h} x^{T}(s)\left(\sum_{j=1}^{N} \gamma_{i j} Q_{3 j}-U_{2}\right) x(s) d s .
\end{aligned}
$$

Likewise, by applying Jensen inequality of Lemma 3 and reciprocally convex combination method of Lemma 5 , the upper bound for the two definite integrals in $\mathscr{L} V_{3}\left(x_{t}, i, t\right)$ can be shown as

$$
\begin{aligned}
-\int_{t-\alpha h}^{t} & \dot{x}^{T}(s) E^{T} R_{2 i} E \dot{x}(s) d s-\int_{t-h}^{t-\alpha h} \dot{x}^{T}(s) E^{T} R_{3 i} E \dot{x}(s) d s \\
= & -\int_{t-h(t)}^{t} \dot{x}^{T}(s) E^{T} R_{2 i} E \dot{x}(s) d s \\
& -\int_{t-\alpha h}^{t-h(t)} \dot{x}^{T}(s) E^{T} R_{2 i} E \dot{x}(s) d s \\
& -\int_{t-h}^{t-\alpha h} \dot{x}^{T}(s) E^{T} R_{3 i} E \dot{x}(s) d s \\
\leq & -\frac{1}{h(t)}(x(t)-x(t-h(t)))^{T} \\
& \times E^{T} R_{2 i} E(x(t)-x(t-h(t))) \\
& -\frac{1}{\alpha h-h(t)}(x(t-h(t))-x(t-\alpha h))^{T} \\
& \times E^{T} R_{2 i} E(x(t-h(t))-x(t-\alpha h)) \\
& -\frac{1}{h-\alpha h}(x(t-\alpha h)-x(t-h))^{T} \\
& \times E^{T} R_{3 i} E(x(t-\alpha h)-x(t-h)) \\
\leq & -\frac{1}{\alpha h}\left[\begin{array}{cc}
x(t)-x(t-h(t)) \\
x(t-h(t))-x(t-\alpha h)
\end{array}\right]^{T} \\
& \times\left[\begin{array}{cc}
E^{T} R_{2 i} E & E^{T} S_{i} E \\
E^{T} S_{i}^{T} E & E^{T} R_{2 i} E
\end{array}\right]\left[\begin{array}{c}
x(t)-x(t-h(t)) \\
x(t-h(t))-x(t-\alpha h)
\end{array}\right]
\end{aligned}
$$

$$
\begin{aligned}
& -\frac{1}{h-\alpha h}(x(t-\alpha h)-x(t-h))^{T} \\
& \times E^{T} R_{3 i} E(x(t-\alpha h)-x(t-h)) \\
= & -\left[\begin{array}{c}
x(t)-x(t-h(t)) \\
x(t-h(t))-x(t-\alpha h) \\
x(t-\alpha h)-x(t-h)
\end{array}\right]^{T} \\
& \times\left[\begin{array}{cc}
\alpha_{1} \bar{E}^{T} Z_{1 i} \bar{E} & 0 \\
0 & \alpha_{2} E^{T} R_{3 i} E
\end{array}\right]\left[\begin{array}{c}
x(t)-x(t-h(t)) \\
x(t-h(t))-x(t-\alpha h) \\
x(t-\alpha h)-x(t-h)
\end{array}\right] .
\end{aligned}
$$

Combining (35)-(39), it yields that

$$
\begin{aligned}
\mathscr{L} V & \left(x_{t}, i, t\right) \leq \eta^{T}(t) \Psi_{1 i} \eta(t) \\
& +\int_{t-h(t)}^{t} x^{T}(s)\left(\sum_{j=1}^{N} \gamma_{i j} Q_{1 j}+\sum_{j=1}^{N} \gamma_{i j} Q_{2 j}-U_{1}\right) x(s) d s \\
& +\int_{-\alpha h}^{0} \int_{t+\theta}^{t} \dot{x}^{T}(s) E^{T}\left(\sum_{j=1}^{N} \gamma_{i j} R_{2 j}-W\right) E \dot{x}(s) d s d \theta \\
& +\int_{t-\alpha h}^{t-h(t)} x^{T}(s)\left(\sum_{j=1}^{N} \gamma_{i j} Q_{2 j}-U_{1}\right) x(s) d s \\
& +\int_{-h}^{-\alpha h} \int_{t+\theta}^{t} \dot{x}^{T}(s) E^{T}\left(\sum_{j=1}^{N} \gamma_{i j} R_{3 j}-W\right) E \dot{x}(s) d s d \theta \\
& +\int_{t-h}^{t-\alpha h} x^{T}(s)\left(\sum_{j=1}^{N} \gamma_{i j} Q_{3 j}-U_{2}\right) x(s) d s,
\end{aligned}
$$

where $\eta^{T}(t)=\left[\begin{array}{llll}x^{T}(t) & x^{T}(t-h(t)) & x^{T}(t-\alpha h) & x^{T}(t-h)\end{array}\right]$, and

$$
\begin{aligned}
\Psi_{1 i}= & \Psi_{i}-\left[\begin{array}{cccc}
I & -I & 0 & 0 \\
0 & I & -I & 0 \\
0 & 0 & I & -I
\end{array}\right]^{T}\left[\begin{array}{cc}
\alpha_{1} \bar{E}^{T} Z_{1 i} \bar{E} & 0 \\
0 & \alpha_{2} E^{T} R_{3 i} E
\end{array}\right] \\
& \times\left[\begin{array}{cccc}
I & -I & 0 & 0 \\
0 & I & -I & 0 \\
0 & 0 & I & -I
\end{array}\right]
\end{aligned}
$$

where $\bar{E}=\operatorname{diag}\{E, E\}$ and

$$
\begin{aligned}
\Psi_{i}= & {\left[\begin{array}{cccc}
\Psi_{i 11} & \Psi_{i 12} & 0 & 0 \\
* & \Psi_{i 22} & 0 & 0 \\
* & * & \Psi_{i 33} & 0 \\
* & * & * & \Psi_{i 44}
\end{array}\right], } \\
\Psi_{i 11}= & A_{i}^{T}\left(P_{i} E+U^{T} \Phi_{i} V^{T}\right)+\left(P_{i} E+U^{T} \Phi_{i} V^{T}\right)^{T} A_{i} \\
& +\sum_{j=1}^{N} \gamma_{i j} E^{T} P_{j} E+Q_{1 i}+Q_{2 i}+\alpha h U_{1}+(1-\alpha) h U_{2} \\
& +A_{i}^{T}\left(\alpha h R_{2 i}+(1-\alpha) h R_{3 i}+\frac{1}{2} h^{2} W\right) A_{i}, \\
\Psi_{i 12}= & \left(P_{i} E+U^{T} \Phi_{i} V^{T}\right)^{T} A_{d i} \\
& +A_{i}^{T}\left(\alpha h R_{2 i}+(1-\alpha) h R_{3 i}+\frac{1}{2} h^{2} W\right) A_{d i},
\end{aligned}
$$




$$
\begin{aligned}
\Psi_{i 22}= & -\left(1-h_{d}\right) Q_{1 i} \\
& +A_{d i}^{T}\left(\alpha h R_{2 i}+(1-\alpha) h R_{3 i}+\frac{1}{2} h^{2} W\right) A_{d i}, \\
\Psi_{i 33}= & Q_{3 i}-Q_{2 i}, \\
\Psi_{i 44}= & -Q_{3 i} .
\end{aligned}
$$

From the Schur complement lemma of Lemma 5, it can be easily verified that $\Psi_{1 i}<0$ holds if (22) holds for $0<h(t) \leq$ $\alpha h$.

Case $2(\alpha h<h(t) \leq h)$. The upper bound can be evaluated for the last five integral terms in $\mathscr{L} V_{2}\left(x_{t}, i, t\right)$, which can be shown as

$$
\begin{aligned}
\int_{t-h(t)}^{t} & x^{T}(s)\left(\sum_{j=1}^{N} \gamma_{i j} Q_{1 j}\right) x(s) d s \\
& +\int_{t-\alpha h}^{t} x^{T}(s)\left(\sum_{j=1}^{N} \gamma_{i j} Q_{2 j}\right) x(s) d s \\
& +\int_{t-h}^{t-\alpha h} x^{T}(s)\left(\sum_{j=1}^{N} \gamma_{i j} Q_{3 j}\right) x(s) d s \\
& -\int_{t-\alpha h}^{t} x^{T}(s) U_{1} x(s) d s-\int_{t-h}^{t-\alpha h} x^{T}(s) U_{2} x(s) d s \\
= & \int_{t-\alpha h}^{t} x^{T}(s)\left(\sum_{j=1}^{N} \gamma_{i j} Q_{1 j}+\sum_{j=1}^{N} \gamma_{i j} Q_{2 j}-U_{1}\right) x(s) d s \\
& +\int_{t-h}^{t-h(t)} x^{T}(s)\left(\sum_{j=1}^{N} \gamma_{i j} Q_{3 j}-U_{2}\right) x(s) d s \\
& +\int_{t-h(t)}^{t-\alpha h} x^{T}(s)\left(\sum_{j=1}^{N} \gamma_{i j} Q_{1 j}+\sum_{j=1}^{N} \gamma_{i j} Q_{3 j}-U_{2}\right) x(s) d s .
\end{aligned}
$$

Likewise, by applying Jensen inequality of Lemma 3 and reciprocally convex combination method of Lemma 5 , the upper bound for the two definite integrals in $\mathscr{L} V_{3}\left(x_{t}, i, t\right)$ can be shown as

$$
\begin{aligned}
- & \int_{t-\alpha h}^{t} \dot{x}^{T}(s) E^{T} R_{2 i} E \dot{x}(s) d s-\int_{t-h}^{t-\alpha h} \dot{x}^{T}(s) E^{T} R_{3 i} E \dot{x}(s) d s \\
= & -\int_{t-\alpha h}^{t} \dot{x}^{T}(s) E^{T} R_{2 i} E \dot{x}(s) d s \\
& -\int_{t-h(t)}^{t-\alpha h} \dot{x}^{T}(s) E^{T} R_{3 i} E \dot{x}(s) d s \\
& -\int_{t-h}^{t-h(t)} \dot{x}^{T}(s) E^{T} R_{3 i} E \dot{x}(s) d s \\
\leq & -\frac{1}{\alpha h}(x(t)-x(t-\alpha h))^{T} \\
& \times E^{T} R_{2 i} E(x(t)-x(t-\alpha h)) \\
& -\frac{1}{h(t)-\alpha h}(x(t-\alpha h)-x(t-h(t)))^{T}
\end{aligned}
$$

$$
\begin{aligned}
& \times E^{T} R_{3 i} E(x(t-\alpha h)-x(t-h(t))) \\
& -\frac{1}{h-h(t)}(x(t-h(t))-x(t-h))^{T} \\
& \times E^{T} R_{3 i} E(x(t-h(t))-x(t-h)) \\
\leq & -\frac{1}{\alpha h}(x(t)-x(t-\alpha h))^{T} \\
& \times E^{T} R_{2 i} E(x(t)-x(t-\alpha h)) \\
& -\frac{1}{h-\alpha h}\left[\begin{array}{cc}
x(t-\alpha h)-x(t-h(t))]^{T} \\
x(t-h(t))-x(t-h)
\end{array}\right]^{T} \\
& \times\left[\begin{array}{cc}
E^{T} R_{3 i} E & E^{T} T_{i} E \\
E^{T} T_{i}^{T} E & E^{T} R_{3 i} E
\end{array}\right]\left[\begin{array}{c}
x(t-\alpha h)-x(t-h(t))-x(t-h) \\
x(t)
\end{array}\right] \\
= & -\left[\begin{array}{c}
x(t)-x(t-\alpha h) \\
x(t-\alpha h)-x(t-h(t)) \\
x(t-h(t))-x(t-h)
\end{array}\right]\left[\begin{array}{cc}
\alpha_{1} E^{T} R_{2 i} E \\
0
\end{array}\right] \\
& \times\left[\begin{array}{c}
x(t)-x(t-\alpha h) \\
x(t-\alpha h)-x(t-h(t)) \\
x(t-h(t))-x(t-h)
\end{array}\right] .
\end{aligned}
$$

Combining (35)-(37) and (43)-(44), it yields that

$$
\begin{aligned}
\mathscr{L} V( & \left.x_{t}, i, t\right) \leq \eta^{T}(t) \Psi_{2 i} \eta(t) \\
& +\int_{t-\alpha h}^{t} x^{T}(s)\left(\sum_{j=1}^{N} \gamma_{i j} Q_{1 j}+\sum_{j=1}^{N} \gamma_{i j} Q_{2 j}-U_{1}\right) x(s) d s \\
& +\int_{t-h(t)}^{t-\alpha h} x^{T}(s)\left(\sum_{j=1}^{N} \gamma_{i j} Q_{1 j}+\sum_{j=1}^{N} \gamma_{i j} Q_{3 j}-U_{2}\right) x(s) d s \\
& +\int_{t-h}^{t-h(t)} x^{T}(s)\left(\sum_{j=1}^{N} \gamma_{i j} Q_{3 j}-U_{2}\right) x(s) d s \\
& +\int_{-\alpha h}^{0} \int_{t+\theta}^{t} \dot{x}^{T}(s) E^{T}\left(\sum_{j=1}^{N} \gamma_{i j} R_{2 j}-W\right) E \dot{x}(s) d s d \theta \\
& +\int_{-h}^{-\alpha h} \int_{t+\theta}^{t} \dot{x}^{T}(s) E^{T}\left(\sum_{j=1}^{N} \gamma_{i j} R_{3 j}-W\right) E \dot{x}(s) d s d \theta
\end{aligned}
$$

where $\eta^{T}(t)=\left[\begin{array}{llll}x^{T}(t) & x^{T}(t-h(t)) & x^{T}(t-\alpha h) & x^{T}(t-h)\end{array}\right]$, and

$$
\begin{aligned}
\Psi_{2 i}= & \Psi_{i}-\left[\begin{array}{cccc}
I & 0 & -I & 0 \\
0 & -I & I & 0 \\
0 & I & 0 & -I
\end{array}\right]^{T}\left[\begin{array}{cc}
\alpha_{1} E^{T} R_{2 i} E & 0 \\
0 & \alpha_{2} \bar{E}^{T} Z_{2 i} \bar{E}
\end{array}\right] \\
& \times\left[\begin{array}{cccc}
I & 0 & -I & 0 \\
0 & -I & I & 0 \\
0 & I & 0 & -I
\end{array}\right] .
\end{aligned}
$$

From the Schur complement lemma of Lemma 5, it can be easily verified that $\Psi_{2 i}<0$ holds if (23) holds for $\alpha h<$ $h(t) \leq h$.

Based on the above analysis, we can know that there exists a scalar $\lambda>0$ such that for each $i \in \ell$

$$
\mathscr{L} V\left(x_{t}, i, t\right) \leq-\lambda\|x(t)\|^{2} .
$$


Then, by Dynkin's formula, we can have for each $i \in \ell$ and $t \geq-h$

$$
\mathscr{E} V\left(x_{t}, i, t\right) \leq \mathscr{E} V\left(x_{0}, i, 0\right)-\lambda \mathscr{E}\|x(t)\|^{2},
$$

which implies that

$$
\mathscr{E}\|x(t)\|^{2} \leq \lambda^{-1} \mathscr{E} V\left(x_{0}, i, 0\right) .
$$

Following a similar line in [12], we can find that the system (4) is stochastically stable under the condition that $w(t)=0$. By Definition 1, the system (4) is stochastically admissible.

In the following, we will establish the $H_{\infty}$ performance index of the system (4). Using the stochastic Lyapunov functional (33), under the zero initial condition, it is from (22)-(27) that

$$
\begin{aligned}
J_{z w}(t) & \\
= & \mathscr{E}\left\{\int_{0}^{t}\left[z^{T}(s) z(s)-\gamma^{2} w^{T}(s) w(s)\right] d s\right\} \\
= & \mathscr{E}\left\{\int_{0}^{t}\left[z^{T}(s) z(s)-\gamma^{2} w^{T}(s) w(s)+\mathscr{L} V\left(x_{s}, i, s\right)\right] d s\right\} \\
& -\mathscr{E} V\left(x_{t}, i, t\right) \\
\leq & \mathscr{E}\left\{\int_{0}^{t}\left[z^{T}(s) z(s)-\gamma^{2} w^{T}(s) w(s)+\mathscr{L} V\left(x_{s}, i, s\right)\right] d s\right\} \\
\leq & \mathscr{E}\left\{\int_{0}^{t} \hat{\eta}^{T}(s) \Theta_{i} \hat{\eta}(s) d s\right\},
\end{aligned}
$$

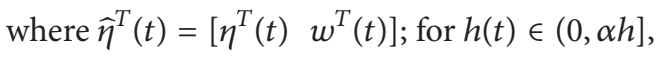

$$
\begin{aligned}
\Theta_{i}= & {\left[\begin{array}{ccccc}
\Theta_{i 11}+C_{i}^{T} C_{i} & \Theta_{i 12}+C_{i}^{T} C_{d i} & \Theta_{i 13} & 0 & \Theta_{i 15}+C_{i}^{T} D_{w i} \\
* & \Theta_{i 22}+C_{d i}^{T} C_{d i} & \Theta_{i 23} & 0 & C_{d i}^{T} D_{w i} \\
* & * & \Theta_{i 33} & \Theta_{i 34} & 0 \\
* & * & * & \Theta_{i 44} & 0 \\
* & * & * & * & D_{w i}^{T} D_{w i}-\gamma^{2} I
\end{array}\right] } \\
& +\left[\begin{array}{c}
A_{i}^{T} \\
A_{d i}^{T} \\
0 \\
0 \\
B_{w i}^{T}
\end{array}\right]\left(\alpha h R_{2 i}+(1-\alpha) h R_{3 i}+\frac{1}{2} h^{2} W\right)\left[\begin{array}{c}
A_{i}^{T} \\
A_{d i}^{T} \\
0 \\
0 \\
B_{w \mathrm{i}}^{T}
\end{array}\right],
\end{aligned}
$$

and for $h(t) \in(\alpha h, h]$,

$$
\begin{aligned}
\Theta_{i}= & {\left[\begin{array}{ccccc}
\Theta_{i 11}+C_{i}^{T} C_{i} & \widehat{\Theta}_{i 12}+C_{i}^{T} C_{d i} & \widehat{\Theta}_{i 13} & 0 & \Theta_{i 15}+C_{i}^{T} D_{w i} \\
* & \widehat{\Theta}_{i 22}+C_{d i}^{T} C_{d i} & \widehat{\Theta}_{i 23} & \widehat{\Theta}_{i 24} & C_{d i}^{T} D_{w i} \\
* & * & \Theta_{i 33} & \widehat{\Theta}_{i 34} & 0 \\
* & * & * & \Theta_{i 44} & 0 \\
* & * & * & * & D_{w i}^{T} D_{w i}-\gamma^{2} I
\end{array}\right] } \\
& +\left[\begin{array}{c}
A_{\dot{j}}^{T} \\
A_{d i}^{T} \\
0 \\
0 \\
B_{w i}^{T}
\end{array}\right]\left(\alpha h R_{2 i}+(1-\alpha) h R_{3 i}+\frac{1}{2} h^{2} W\right)\left[\begin{array}{c}
A_{i}^{T} \\
A_{d i}^{T} \\
0 \\
0 \\
B_{w i}^{T}
\end{array}\right]^{T}
\end{aligned}
$$

By Schur complement lemma of Lemma 6, together with (22)-(27), we have that $J_{z w}(t)<0$ for all $t>0$. Consequently, under the zero initial condition, we have that (6) holds for any nonzero $w(t) \in \mathscr{L}_{2}[0, \infty)$. Therefore, by Definition 2, the descriptor Markovian jump time-delay system (4) is stochastically admissible with $H_{\infty}$ performance level $\gamma$ for any time delay $h(t)$ satisfying (3). This completes the proof.

Remark 10. By virtue of the novel Lyapunov-Krasovskii functional, Theorem 9 provides a delay-dependent and delayderivative-dependent $H_{\infty}$ criterion to guarantee the system (4) to be stochastically admissible with $H_{\infty}$ performance level. The novelty of Theorem 9 lies in the new LyapunovKrasovskii functional and the reciprocally convex combination method. By dividing the delay interval $(0, h]$ into two subintervals $(0, \alpha h]$ and $(\alpha h, h]$ the following modedependent definite integrals

$$
\int_{t-\alpha h}^{t} x^{T}(s) Q_{2}\left(r_{t}\right) x(s) d s+\int_{t-h}^{t-\alpha h} x^{T}(s) Q_{3}\left(r_{t}\right) x(s) d s
$$

and mode-dependent double integrals

$$
\begin{aligned}
& \int_{-\alpha h}^{0} \int_{t+\theta}^{t} \dot{x}^{T}(s) E^{T} R_{2}\left(r_{t}\right) E \dot{x}(s) d s d \theta \\
& \quad+\int_{-h}^{-\alpha h} \int_{t+\theta}^{t} \dot{x}^{T}(s) E^{T} R_{3}\left(r_{t}\right) E \dot{x}(s) d s d \theta
\end{aligned}
$$

are introduced. Obviously, the above integral terms take the mode $r_{t}$ into full consideration and contain the modeindependent one [12] as a special case. Since $h(t)$ is restricted to the subinterval $(0, \alpha h]$ or $(\alpha h, h]$ and various LyapunovKrasovskii functionals are selected for different subsystems, our method can lead to reduction of conservatism.

For the unforced system (4), if disturbance signal $w(t) \equiv$ 0 , then we have the following time-delay systems:

$$
\begin{aligned}
E \dot{x}(t) & =A\left(r_{t}\right) x(t)+A_{d}\left(r_{t}\right) x(t-h(t)) x(\theta) \\
& =\phi(\theta), \quad \forall \theta \in[-h, 0] .
\end{aligned}
$$

Based on Theorem 9, the following stochastic admissibility result can be given.

Corollary 11. For given scalars $h>0,0 \leq h_{d}<1$, the system (55) is stochastically admissible if there exist a set of positive definite matrices $P_{i}, Q_{1 i}, Q_{2 i}, Q_{3 i}, R_{2 i}, R_{3 i}, U_{1}, U_{2}, W$ and matrices $\Phi_{i}, S_{i}, T_{i}, \forall i \in \ell$, satisfying the following LMIs for each $i \in \ell$ :

$$
\begin{aligned}
\Theta_{1 i}= & \Theta_{i}-\left[\begin{array}{ccccc}
I & -I & 0 & 0 & 0 \\
0 & I & -I & 0 & 0 \\
0 & 0 & I & -I & 0 \\
0 & 0 & 0 & 0 & I
\end{array}\right]^{T} \\
& \times\left[\begin{array}{cccc}
\alpha_{1} \bar{E}^{T} Z_{1 i} \bar{E} & 0 & 0 \\
0 & \alpha_{2} E^{T} R_{3 i} E & 0 \\
0 & 0 & 0
\end{array}\right]\left[\begin{array}{ccccc}
I & -I & 0 & 0 & 0 \\
0 & I & -I & 0 & 0 \\
0 & 0 & I & -I & 0 \\
0 & 0 & 0 & 0 & I
\end{array}\right] \\
& <0,
\end{aligned}
$$




$$
\begin{gathered}
\Theta_{2 i}=\Theta_{i}-\left[\begin{array}{ccccc}
I & 0 & -I & 0 & 0 \\
0 & -I & I & 0 & 0 \\
0 & I & 0 & -I & 0 \\
0 & 0 & 0 & 0 & I
\end{array}\right]^{T} \\
\times\left[\begin{array}{ccc}
\alpha_{1} E^{T} R_{2 i} E & 0 & 0 \\
0 & \alpha_{2} \bar{E}^{T} Z_{2 i} \bar{E} & 0 \\
0 & 0 & 0
\end{array}\right]\left[\begin{array}{ccccc}
I & 0 & -I & 0 & 0 \\
0 & -I & I & 0 & 0 \\
0 & I & 0 & -I & 0 \\
0 & 0 & 0 & 0 & I
\end{array}\right] \\
<0, \quad \sum_{j=1}^{N} \gamma_{i j} R_{3 j}-W<0, \\
\sum_{j=1}^{N} \gamma_{i j} Q_{1 j}+\sum_{j=1}^{N} \gamma_{i j} Q_{2 j}-U_{1}<0, \quad \sum_{j=1}^{N} \gamma_{i j} Q_{2 j}-U_{1}<0 \\
\sum_{j=1}^{N} \gamma_{i j} Q_{1 j}+\sum_{j=1}^{N} \gamma_{i j} Q_{3 j}-U_{2}<0, \quad \sum_{j=1}^{N} \gamma_{i j} Q_{3 j}-U_{2}<0
\end{gathered}
$$

where $\alpha_{1}=1 / \alpha h, \alpha_{2}=1 /(h-\alpha h), \bar{E}=\operatorname{diag}\{E, E\}$, and

$$
\begin{aligned}
\Theta_{i}= & {\left[\begin{array}{ccccc}
\Theta_{i 11} & \Theta_{i 12} & 0 & 0 & \Theta_{i 15} \\
* & \Theta_{i 22} & 0 & 0 & \Theta_{i 25} \\
* & * & \Theta_{i 33} & 0 & 0 \\
* & * & * & \Theta_{i 44} & 0 \\
* & * & * & * & \Theta_{i 55}
\end{array}\right] } \\
\Theta_{i 11}= & A_{i}^{T}\left(P_{i} E+U^{T} \Phi_{i} V^{T}\right)+\left(P_{i} E+U^{T} \Phi_{i} V^{T}\right)^{T} A_{i} \\
& +\sum_{j=1}^{N} \gamma_{i j} E^{T} P_{j} E+Q_{1 i}+Q_{2 i}+\alpha h U_{1} \\
& +(1-\alpha) h U_{2}, \\
\Theta_{i 12}= & \left(P_{i} E+U^{T} \Phi_{i} V^{T}\right)^{T} A_{d i}, \\
\Theta_{i 15}= & A_{i}^{T}\left(\alpha h R_{2 i}+(1-\alpha) h R_{3 i}+\frac{1}{2} h^{2} W\right) \\
\Theta_{i 22}= & -\left(1-h_{d}\right) Q_{1 i}, \\
\Theta_{i 25}= & A_{d i}^{T}\left(\alpha h R_{2 i}+(1-\alpha) h R_{3 i}+\frac{1}{2} h^{2} W\right) \\
\Theta_{i 33}= & Q_{3 i}-Q_{2 i}, \\
\Theta_{i 44}= & -Q_{3 i}, \\
\Theta_{i 55}= & -\left(\alpha h R_{2 i}+(1-\alpha) h R_{3 i}+\frac{1}{2} h^{2} W\right) .
\end{aligned}
$$

When the time delay is time-invariant, the system (55) reduces to the one

$$
\begin{aligned}
E \dot{x}(t) & =A\left(r_{t}\right) x(t)+A_{d}\left(r_{t}\right) x(t-h) x(\theta) \\
& =\phi(\theta), \quad \forall \theta \in[-h, 0] .
\end{aligned}
$$

Using the similar method of Corollary 11, we have the stochastic admissibility condition for (59).
Corollary 12. For given scalars $h>0$, the system (59) is stochastically admissible if there exist a set of positive definite matrices $P_{i}, Q_{2 i}, Q_{3 i}, R_{2 i}, R_{3 i}, U_{1}, U_{2}, W$ and matrices $\Phi_{i}$, $\forall i \in \ell$, satisfying the following LMIs for each $i \in \ell$ :

$$
\begin{aligned}
\bar{\Theta}_{i}= & \Theta_{i}-\left[\begin{array}{cccc}
I & -I & 0 & 0 \\
0 & I & -I & 0 \\
0 & 0 & 0 & I
\end{array}\right]^{T}\left[\begin{array}{ccc}
\alpha_{1} E^{T} R_{2 i} E & 0 & 0 \\
0 & \alpha_{2} E^{T} R_{3 i} E & 0 \\
0 & 0 & 0
\end{array}\right] \\
& \times\left[\begin{array}{cccc}
I & -I & 0 & 0 \\
0 & I & -I & 0 \\
0 & 0 & 0 & I
\end{array}\right]<0, \\
& \sum_{j=1}^{N} \gamma_{i j} R_{2 j}-W<0, \quad \sum_{j=1}^{N} \gamma_{i j} R_{3 j}-W<0, \\
& \sum_{j=1}^{N} \gamma_{i j} Q_{2 j}-U_{1}<0, \quad \sum_{j=1}^{N} \gamma_{i j} Q_{3 j}-U_{2}<0,
\end{aligned}
$$

where $\alpha_{1}=1 / \alpha h, \alpha_{2}=1 /(h-\alpha h)$; and

$$
\begin{aligned}
\Theta_{i}= & {\left[\begin{array}{cccc}
\Theta_{i 11} & 0 & \Theta_{i 13} & \Theta_{i 14} \\
* & \Theta_{i 22} & 0 & 0 \\
* & * & \Theta_{i 33} & \Theta_{i 34} \\
* & * & * & \Theta_{i 44}
\end{array}\right], } \\
\Theta_{i 11}= & A_{i}^{T}\left(P_{i} E+U^{T} \Phi_{i} V^{T}\right)+\left(P_{i} E+U^{T} \Phi_{i} V^{T}\right)^{T} A_{i} \\
& +\sum_{j=1}^{N} \gamma_{i j} E^{T} P_{j} E+Q_{2 i}+\alpha h U_{1}+(1-\alpha) h U_{2}, \\
\Theta_{i 13}= & \left(P_{i} E+U^{T} \Phi_{i} V^{T}\right)^{T} A_{d i}, \\
\Theta_{i 14}= & A_{i}^{T}\left(\alpha h R_{2 i}+(1-\alpha) h R_{3 i}+\frac{1}{2} h^{2} W\right), \\
\Theta_{i 22}= & Q_{3 i}-Q_{2 i}, \\
\Theta_{i 33}= & -Q_{3 i}, \\
\Theta_{i 34}= & A_{d i}^{T}\left(\alpha h R_{2 i}+(1-\alpha) h R_{3 i}+\frac{1}{2} h^{2} W\right), \\
\Theta_{i 44}= & -\left(\alpha h R_{2 i}+(1-\alpha) h R_{3 i}+\frac{1}{2} h^{2} W\right) .
\end{aligned}
$$

Remark 13. By decomposing the delay interval $(0, h]$ into two segments $(0, \alpha h]$ and $(\alpha h, h]$, Theorem 9, Corollary 11, and Corollary 12 give delay-dependent conditions for the underlying systems with time-varying and time-invariant delay, respectively. The algorithm about how to select tuning parameter $\alpha$ optimally can be referred to the algorithm in the end of this section.

Now, we are ready to deal with the delay-dependent $H_{\infty}$ control problem for the descriptor Markovian jump systems (1). For this purpose, applying the controller in (7) to the system (1), then we can have the following closed-loop systems:

$$
\begin{aligned}
E \dot{x}(t)= & \left(A\left(r_{t}\right)+B\left(r_{t}\right) K\left(r_{t}\right)\right) x(t) \\
& +\left(A_{d}\left(r_{t}\right)+B\left(r_{t}\right) K_{d}\left(r_{t}\right)\right) x(t-h(t)) \\
& +B_{w}\left(r_{t}\right) w(t)
\end{aligned}
$$




$$
\begin{aligned}
z(t)= & \left(C\left(r_{t}\right)+D\left(r_{t}\right) K\left(r_{t}\right)\right) x(t) \\
& +\left(C_{d}\left(r_{t}\right)+D\left(r_{t}\right) K_{d}\left(r_{t}\right)\right) x(t-h(t)) \\
& +D_{w}\left(r_{t}\right) w(t), \\
& x(\theta)=\phi(\theta), \quad \forall \theta \in[-h, 0] .
\end{aligned}
$$

On the basis of Theorem 9, the following result, which provides the design method of state feedback controller and ensures the closed-loop systems (3) to be stochastically admissible with $H_{\infty}$ performance level $\gamma$, can be obtained.

Theorem 14. For given scalars $\gamma>0, \varepsilon_{i}>0, h>0$, and $0 \leq h_{d}<1$, the descriptor Markovian jump time-delay system (1) is stochastically admissible with $H_{\infty}$ performance level $\gamma$ for any $h(t)$ satisfying (3), if there exist a set of positive definite matrices $\bar{P}_{i}, \bar{Q}_{1 i j}, \bar{Q}_{2 i j}, \bar{Q}_{3 i j}, \bar{R}_{2 i}, \bar{R}_{3 i}, \bar{U}_{1 i}, \bar{U}_{2 i}, \bar{W}$ and matrices $\bar{\Phi}_{i}, H_{i}, L_{i}, \forall i \in \ell, j \in \ell$, satisfying the following LMIs for each $i \in \ell$ :

$$
\begin{aligned}
& \left.\left[\begin{array}{ccccccccc}
\Upsilon_{i 11} & \breve{\Upsilon}_{i 12} & 0 & 0 & B_{w i} & \Upsilon_{i 16} & \frac{1}{2} h^{2}\left(A_{i} \Lambda_{i}+B_{i} H_{i}\right)^{T} & \left(C_{i} \Lambda_{i}+D_{i} H_{i}\right)^{T} & \Upsilon_{i 19} \\
* & \breve{\Upsilon}_{i 22} & \breve{\Upsilon}_{i 23} & 0 & 0 & \Upsilon_{i 26} & \frac{1}{2} h^{2}\left(A_{d i} \Lambda_{i}+B_{i} L_{i}\right)^{T} & \left(C_{d i} \Lambda_{i}+D_{i} L_{i}\right)^{T} & 0 \\
* & * & \Upsilon_{i 33} & \breve{\Upsilon}_{i 34} & 0 & 0 & 0 & 0 & 0 \\
* & * & * & \Upsilon_{i 44} & 0 & 0 & 0 & 0 & 0 \\
* & * & * & * & -\gamma^{2} I & \Upsilon_{i 56} & \frac{1}{2} h^{2} B_{w i}^{T} & D_{w i}^{T} & 0 \\
* & * & * & * & * & \Upsilon_{i 66} & 0 & 0 & 0 \\
* & * & * & * & * & * & -\frac{1}{2} h^{2} \bar{W} & 0 & 0 \\
* & * & * & * & * & * & * & * & 0 \\
* & * & * & * & * & * & * & * & \Upsilon_{i 99}
\end{array}\right]<0, \alpha h\right], \\
& {\left[\begin{array}{ccccccccc}
\Upsilon_{i 11} & \widehat{\Upsilon}_{i 12} & \widehat{\Upsilon}_{i 13} & 0 & B_{w i} & \Upsilon_{i 16} & \frac{1}{2} h^{2}\left(A_{i} \Lambda_{i}+B_{i} H_{i}\right)^{T} & \left(C_{i} \Lambda_{i}+D_{i} H_{i}\right)^{T} & \Upsilon_{i 19} \\
* & \widehat{\Upsilon}_{i 22} & \widehat{\Upsilon}_{i 23} & \widehat{\Upsilon}_{i 24} & 0 & \Upsilon_{i 26} & \frac{1}{2} h^{2}\left(A_{d i} \Lambda_{i}+B_{i} L_{i}\right)^{T} & \left(C_{d i} \Lambda_{i}+D_{i} L_{i}\right)^{T} & 0 \\
* & * & \Upsilon_{i 33} & 0 & 0 & 0 & 0 & 0 & 0 \\
* & * & * & \Upsilon_{i 44} & 0 & 0 & 0 & 0 & 0 \\
* & * & * & * & -\gamma^{2} I & \Upsilon_{i 56} & \frac{1}{2} h^{2} B_{w i}^{T} & D_{w i}^{T} & 0 \\
* & * & * & * & * & \Upsilon_{i 66} & 0 & 0 & 0 \\
* & * & * & * & * & * & -\frac{1}{2} h^{2} \bar{W} & 0 & 0 \\
* & * & * & * & * & * & * & -I & 0 \\
* & * & * & * & * & * & * & * & \Upsilon_{i 99}
\end{array}\right]<0, \quad h(t) \in(\alpha h, h],} \\
& \gamma_{i j}(N-1) \bar{W}<\bar{R}_{2 j}, \quad \gamma_{i j}(N-1) \bar{W}<\bar{R}_{3 j}, \quad \forall j \neq i, \quad \gamma_{i j} \neq 0, \\
& \sum_{j=1}^{N} \gamma_{i j} \bar{Q}_{1 i j}+\sum_{j=1}^{N} \gamma_{i j} \bar{Q}_{2 i j}-\bar{U}_{1 i}<0, \quad \sum_{j=1}^{N} \gamma_{i j} \bar{Q}_{2 i j}-\bar{U}_{1 i}<0, \\
& \sum_{j=1}^{N} \gamma_{i j} \bar{Q}_{1 i j}+\sum_{j=1}^{N} \gamma_{i j} \bar{Q}_{3 i j}-\bar{U}_{2 i}<0, \quad \sum_{j=1}^{N} \gamma_{i j} \bar{Q}_{3 i j}-\bar{U}_{2 i}<0,
\end{aligned}
$$

where $\alpha_{1}=1 / \alpha h, \alpha_{2}=1 /(h-\alpha h), \Lambda_{i}=\bar{P}_{i} E^{T}+V \bar{\Phi}_{i} U$, and

$$
\begin{aligned}
\Upsilon_{i 11}= & \Lambda_{i}^{T} A_{i}^{T}+H_{i}^{T} B_{i}^{T}+A_{i} \Lambda_{i}+B_{i} H_{i} \\
& +\gamma_{i i}\left(\epsilon_{i} \Lambda_{i}^{T} E^{T}+\epsilon_{i} E \Lambda_{i}-\epsilon_{i}^{2} E \bar{P}_{i} E^{T}\right) \\
& +\bar{Q}_{1 i i}+\bar{Q}_{2 i i}+\alpha h \bar{U}_{1 i}+(1-\alpha) h \bar{U}_{2 i} \\
& -\Lambda_{i}^{T} E^{T}-E \Lambda_{i}+\alpha h \bar{R}_{2 i}, \\
\breve{\Upsilon}_{i 12}= & A_{d i} \Lambda_{i}+B_{i} L_{i}+\Lambda_{i}^{T} E^{T}+E \Lambda_{i}-\alpha h \bar{R}_{2 i}, \\
\Upsilon_{i 16}= & {\left[\left(A_{i} \Lambda_{i}+B_{i} H_{i}\right)^{T}\left(A_{i} \Lambda_{i}+B_{i} H_{i}\right)^{T}\right], }
\end{aligned}
$$

$$
\begin{aligned}
& \Upsilon_{i 19} \\
& =\left[\sqrt{\gamma_{i 1}} \Lambda_{i}^{T} E_{R} \cdots \sqrt{\gamma_{i(i-1)}} \Lambda_{i}^{T} E_{R} \sqrt{\gamma_{i(i+1)}} \Lambda_{i}^{T} E_{R} \cdots \sqrt{\gamma_{i N}} \Lambda_{i}^{T} E_{R}\right], \\
& \breve{\Upsilon}_{i 22}=-\left(1-h_{d}\right) \bar{Q}_{1 i i}-2 \Lambda_{i}^{T} E^{T}-2 E \Lambda_{i}+2 \alpha h \bar{R}_{2 i}, \\
& \breve{\Upsilon}_{i 23}=\Lambda_{i}^{T} E^{T}+E \Lambda_{i}-\alpha h \bar{R}_{2 i} \\
& \Upsilon_{i 26}=\left[\left(A_{d i} \Lambda_{i}+B_{i} L_{i}\right)^{T}\left(A_{d i} \Lambda_{i}+B_{i} L_{i}\right)^{T}\right] \\
& \Upsilon_{i 33}=\bar{Q}_{3 i i}-\bar{Q}_{2 i i}-2 \Lambda_{i}^{T} E^{T}-2 E \Lambda_{i}+\alpha h \bar{R}_{2 i}+(h-\alpha h) \bar{R}_{3 i}, \\
& \breve{\Upsilon}_{i 34}=\Lambda_{i}^{T} E^{T}+E \Lambda_{i}-(h-\alpha h) \bar{R}_{3 i},
\end{aligned}
$$


$\Upsilon_{i 44}=-Q_{3 i i}-\Lambda_{i}^{T} E^{T}-E \Lambda_{i}+(h-\alpha h) \bar{R}_{3 i}$,

$\Upsilon_{i 56}=\left[\begin{array}{ll}B_{w i}^{T} & B_{w i}^{T}\end{array}\right]$,

$\Upsilon_{i 66}=\operatorname{diag}\left\{-\alpha_{1} \bar{R}_{2 i},-\alpha_{2} \bar{R}_{3 i}\right\}$,

$\Upsilon_{i 99}$

$=-\operatorname{diag}\left\{E_{R}^{T} \bar{P}_{1} E_{R}, \ldots, E_{R}^{T} \bar{P}_{i-1} E_{R}, E_{R}^{T} \bar{P}_{i+1} E_{R}, \ldots, E_{R}^{T} \bar{P}_{N} E_{R}\right\}$,

$\widehat{\Upsilon}_{i 12}=A_{d i} \Lambda_{i}+B_{i} L_{i}$,

$\widehat{\Upsilon}_{i 13}=\Lambda_{i}^{T} E^{T}+E \Lambda_{i}-\alpha h \bar{R}_{2 i}$

$\widehat{\Upsilon}_{i 22}=-\left(1-h_{d}\right) \bar{Q}_{1 i i}-2 \Lambda_{i}^{T} E^{T}-2 E \Lambda_{i}+2(h-\alpha h) \bar{R}_{3 i}$,

$\widehat{\Upsilon}_{i 23}=\Lambda_{i}^{T} E^{T}+E \Lambda_{i}-(h-\alpha h) \bar{R}_{3 i}$,

$\widehat{Y}_{i 24}=\widehat{Y}_{i 23}$.
Furthermore, if the above LMIs have feasible solutions, then the desired state feedback controller can be solved as $K_{i}=H_{i} \Lambda_{i}^{-1}$, $K_{d i}=L_{i} \Lambda_{i}^{-1}$.

Proof. It follows from Theorem 9 that the closed-loop system (3) is stochastically admissible with $H_{\infty}$ performance level $\gamma$ if there exist a set of matrices such that (22)-(27) hold, where $A_{i}, A_{d i}, C_{i}$, and $C_{d i}$ are replaced by $A_{i}+B_{i} K_{i}, A_{d i}+B_{i} K_{d i}$, $C_{i}+D_{i} K_{i}$, and $C_{d i}+D_{i} K_{d i}$, respectively.

According to Lemma 8, we can set $\Lambda_{i}=\left(P_{i} E+U^{T}\right.$ $\left.\Phi_{i} V^{T}\right)^{-1}=\bar{P}_{i} E^{T}+V \bar{\Phi}_{i} U$; then pre- and postmultiplying (22) and (23) by $\operatorname{diag}\left\{\Lambda_{i}^{T}, \Lambda_{i}^{T}, \Lambda_{i}^{T}, \Lambda_{i}^{T}, I, R_{2 i}^{-T}, R_{3 i}^{-T}, W^{-T}, I\right\}$ and its transpose and leting $H_{i}=K_{i}\left(\bar{P}_{i} E^{T}+V \bar{\Phi}_{i} U\right), L_{i}=K_{d i}\left(\bar{P}_{i} E^{T}+\right.$ $\left.V \bar{\Phi}_{i} U\right)$, we have

$$
\begin{aligned}
& \left.\left[\begin{array}{cccccccc}
\breve{\Theta}_{i 11} & \breve{\Theta}_{i 12} & \breve{\Theta}_{i 13} & 0 & B_{w i} & \breve{\Theta}_{i 16} & \frac{1}{2} h^{2}\left(A_{i} \Lambda_{i}+B_{i} H_{i}\right)^{T} & \left(C_{i} \Lambda_{i}+D_{i} H_{i}\right)^{T} \\
* & \breve{\Theta}_{i 22} & \breve{\Theta}_{i 23} & 0 & 0 & \breve{\Theta}_{i 26} & \frac{1}{2} h^{2}\left(A_{d i} \Lambda_{i}+B_{i} L_{i}\right)^{T} & \left(C_{d i} \Lambda_{i}+D_{i} L_{i}\right)^{T} \\
* & * & \breve{\Theta}_{i 33} & \breve{\Theta}_{i 34} & 0 & 0 & 0 & 0 \\
* & * & * & \breve{\Theta}_{i 44} & 0 & 0 & 0 & 0 \\
* & * & * & * & -\gamma^{2} I & \breve{\Theta}_{i 56} & \frac{1}{2} h^{2} B_{w i}^{T} & D_{w i}^{T} \\
* & * & * & * & * & \breve{\Theta}_{i 66} & 0 & 0 \\
* & * & * & * & * & * & -\frac{1}{2} h^{2} W^{-1} & 0 \\
* & * & * & * & * & * & * & -I
\end{array}\right]<0, \alpha h\right], \\
& {\left[\begin{array}{cccccccc}
\breve{\Theta}_{i 11} & \widehat{\Theta}_{i 12} & \widehat{\Theta}_{i 13} & 0 & B_{w i} & \breve{\Theta}_{i 16} & \frac{1}{2} h^{2}\left(A_{i} \Lambda_{i}+B_{i} H_{i}\right)^{T} & \left(C_{i} \Lambda_{i}+D_{i} H_{i}\right)^{T} \\
* & \widehat{\Theta}_{i 22} & \widehat{\Theta}_{i 23} & \widehat{\Theta}_{i 24} & 0 & \breve{\Theta}_{i 26} & \frac{1}{2} h^{2}\left(A_{d i} \Lambda_{i}+B_{i} L_{i}\right)^{T} & \left(C_{d i} \Lambda_{i}+D_{i} L_{i}\right)^{T} \\
* & * & \breve{\Theta}_{i 33} & \widehat{\Theta}_{i 34} & 0 & 0 & 0 & 0 \\
* & * & * & \breve{\Theta}_{i 44} & 0 & 0 & 0 & 0 \\
* & * & * & * & -\gamma^{2} I & \breve{\Theta}_{i 56} & \frac{1}{2} h^{2} B_{w i}^{T} & D_{w i}^{T} \\
* & * & * & * & * & \breve{\Theta}_{i 66} & 0 & 0 \\
* & * & * & * & * & * & -\frac{1}{2} h^{2} W^{-1} & 0 \\
* & * & * & * & * & * & * & -I
\end{array}\right]<0, \quad h(t) \in(\alpha h, h],}
\end{aligned}
$$

where

$$
\begin{aligned}
\breve{\Theta}_{i 11}= & \Lambda_{i}^{T} A_{i}^{T}+H_{i}^{T} B_{i}^{T}+A_{i} \Lambda_{i}+B_{i} H_{i} \\
& +\Lambda_{i}^{T}\left(\sum_{j=1}^{N} \gamma_{i j} E_{R}\left(E_{R}^{T} \bar{P}_{j} E_{R}\right)^{-1} E_{R}^{T}\right) \Lambda_{i} \\
& +\Lambda_{i}^{T} Q_{1 i} \Lambda_{i}+\Lambda_{i}^{T} Q_{2 i} \Lambda_{i}+\alpha h \Lambda_{i}^{T} U_{1} \Lambda_{i} \\
& +(1-\alpha) h \Lambda_{i}^{T} U_{2} \Lambda_{i}-\alpha_{1} \Lambda_{i}^{T} E^{T} R_{2 i} E \Lambda_{i}, \\
\breve{\Theta}_{i 12}= & A_{d i} \Lambda_{i}+B_{i} L_{i}+\alpha_{1} \Lambda_{i}^{T} E^{T} R_{2 i} E \Lambda_{i}-\alpha_{1} \Lambda_{i}^{T} E^{T} S_{i} E \Lambda_{i},
\end{aligned}
$$

$$
\breve{\Theta}_{i 13}=\alpha_{1} \Lambda_{i}^{T} E^{T} S_{i} E \Lambda_{i},
$$$$
\breve{\Theta}_{i 16}=\left[\left(A_{i} \Lambda_{i}+B_{i} H_{i}\right)^{T}\left(A_{i} \Lambda_{i}+B_{i} H_{i}\right)^{T}\right],
$$$$
\breve{\Theta}_{i 22}=-\left(1-h_{d}\right) \Lambda_{i}^{T} Q_{1 i} \Lambda_{i}-2 \alpha_{1} \Lambda_{i}^{T} E^{T} R_{2 i} E \Lambda_{i} \text {, }
$$$$
+\alpha_{1} \Lambda_{i}^{T} E^{T} S_{i} E \Lambda_{i}+\alpha_{1} \Lambda_{i}^{T} E^{T} S_{i}^{T} E \Lambda_{i} \text {, }
$$$$
\breve{\Theta}_{i 23}=\alpha_{1} \Lambda_{i}^{T} E^{T} R_{2 i} E \Lambda_{i}^{T}-\alpha_{1} \Lambda_{i}^{T} E^{T} S_{i} E \Lambda_{i}^{T} \text {, }
$$$$
\breve{\Theta}_{i 26}=\left[\left(A_{d i} \Lambda_{i}+B_{i} L_{i}\right)^{T}\left(A_{d i} \Lambda_{i}+B_{i} L_{i}\right)^{T}\right],
$$ 


$$
\begin{aligned}
& \breve{\Theta}_{i 33}=\Lambda_{i}^{T} Q_{3 i} \Lambda_{i}-\Lambda_{i}^{T} Q_{2 i} \Lambda_{i} \\
& -\alpha_{1} \Lambda_{i}^{T} E^{T} R_{2 i} E \Lambda_{i}-\Lambda_{i}^{T} \alpha_{2} E^{T} R_{3 i} E \Lambda_{i}, \\
& \widehat{\Theta}_{i 23}=\alpha_{2} \Lambda_{i}^{T} E^{T} R_{3 i} E \Lambda_{i}-\alpha_{2} \Lambda_{i}^{T} E^{T} T_{i}^{T} E \Lambda_{i}, \\
& \widehat{\Theta}_{i 24}=\alpha_{2} \Lambda_{i}^{T} E^{T} R_{3 i} E \Lambda_{i}-\alpha_{2} \Lambda_{i}^{T} E^{T} T_{i} E \Lambda_{i}, \\
& \breve{\Theta}_{i 34}=\alpha_{2} \Lambda_{i}^{T} E^{T} R_{3 i} E \Lambda_{i} \text {, } \\
& \breve{\Theta}_{i 44}=-\Lambda_{i}^{T} Q_{3 i} \Lambda_{i}-\alpha_{2} \Lambda_{i}^{T} E^{T} R_{3 i} E \Lambda_{i} \text {, } \\
& \breve{\Theta}_{i 56}=\left[B_{w i}^{T} B_{w i}^{T}\right] \text {, } \\
& \breve{\Theta}_{i 66}=\operatorname{diag}\left\{-\alpha_{1} R_{2 i}^{-1},-\alpha_{2} R_{3 i}^{-1}\right\} \text {, } \\
& \widehat{\Theta}_{i 12}=A_{d i} \Lambda_{i}+B_{i} L_{i} \text {, } \\
& \widehat{\Theta}_{i 13}=\alpha_{1} \Lambda_{i}^{T} E^{T} R_{2 i} E \Lambda_{i} \text {, } \\
& \widehat{\Theta}_{i 22}=-\left(1-h_{d}\right) \Lambda_{i}^{T} Q_{1 i} \Lambda_{i}-2 \alpha_{2} \Lambda_{i}^{T} E^{T} R_{3 i} E \Lambda_{i} \\
& +\alpha_{2} \Lambda_{i}^{T} E^{T} T_{i} E \Lambda_{i}+\alpha_{1} \Lambda_{i}^{T} E^{T} T_{i}^{T} E \Lambda_{i} \text {, } \\
& \widehat{\Theta}_{i 34}=\alpha_{2} \Lambda_{i}^{T} E^{T} T_{3 i} E \Lambda_{i} \text {. } \\
& \gamma_{i i} \Lambda_{i}^{T} E_{R}\left(E_{R}^{T} \bar{P}_{i} E_{R}\right)^{-1} E_{R}^{T} \Lambda_{i} \\
& \leq \gamma_{i i}\left(\varepsilon_{i} \Lambda_{i}^{T} E^{T}+\varepsilon_{i} E \Lambda_{i}-\varepsilon_{i}^{2} E \bar{P}_{i} E^{T}\right) . \\
& -\left[\begin{array}{cccc}
\alpha_{1} \Lambda_{i}^{T} E^{T} R_{2 i} E \Lambda_{i} & 0 & -\alpha_{1} \Lambda_{i}^{T} E^{T} R_{2 i} E \Lambda_{i} & 0 \\
0 & 2 \alpha_{2} \Lambda_{i}^{T} E^{T} R_{3 i} E \Lambda_{i} & -\alpha_{2} \Lambda_{i}^{T} E^{T} R_{3 i} E \Lambda_{i} & -\alpha_{2} \Lambda_{i}^{T} E^{T} R_{3 i} E \Lambda_{i} \\
-\alpha_{1} \Lambda_{i}^{T} E^{T} R_{2 i} E \Lambda_{i} & -\alpha_{2} \Lambda_{i}^{T} E^{T} R_{3 i} E \Lambda_{i} & \alpha_{1} \Lambda_{i}^{T} E^{T} R_{2 i} E \Lambda_{i}+\alpha_{2} \Lambda_{i}^{T} E^{T} R_{3 i} E \Lambda_{i} & 0 \\
0 & -\alpha_{2} \Lambda_{i}^{T} E^{T} R_{3 i} E \Lambda_{i} & 0 & \alpha_{2} \Lambda_{i}^{T} E^{T} R_{3 i} E \Lambda_{i}
\end{array}\right] \\
& =\left[\begin{array}{c}
I \\
0 \\
-I \\
0
\end{array}\right]\left(-\alpha_{1} \Lambda_{i}^{T} E^{T} R_{2 i} E \Lambda_{i}\right)\left[\begin{array}{llll}
I & 0 & -I & 0
\end{array}\right]+\left[\begin{array}{c}
0 \\
I \\
-I \\
0
\end{array}\right]\left(-\alpha_{2} \Lambda_{i}^{T} E^{T} R_{3 i} E \Lambda_{i}\right)\left[\begin{array}{llll}
0 & I & -I & 0
\end{array}\right] \\
& +\left[\begin{array}{c}
0 \\
I \\
0 \\
-I
\end{array}\right]\left(-\alpha_{2} \Lambda_{i}^{T} E^{T} R_{3 i} E \Lambda_{i}\right)\left[\begin{array}{llll}
0 & I & 0 & -I
\end{array}\right] \leq\left[\begin{array}{c}
I \\
0 \\
-I \\
0
\end{array}\right]\left(-\Lambda_{i}^{T} E^{T}-E \Lambda_{i}+\alpha h R_{2 i}^{-1}\right)\left[\begin{array}{llll}
I & 0 & -I & 0
\end{array}\right] \\
& +\left[\begin{array}{c}
0 \\
I \\
-I \\
0
\end{array}\right]\left(-\Lambda_{i}^{T} E^{T}-E \Lambda_{i}+(h-\alpha h) R_{3 i}^{-1}\right)\left[\begin{array}{llll}
0 & I & -I & 0
\end{array}\right]+\left[\begin{array}{c}
0 \\
I \\
0 \\
-I
\end{array}\right]\left(-\Lambda_{i}^{T} E^{T}-E \Lambda_{i}+(h-\alpha h) R_{3 i}^{-1}\right)\left[\begin{array}{llll}
0 & I & 0 & -I
\end{array}\right], \\
& -\left[\begin{array}{cccc}
\alpha_{1} \Lambda_{i}^{T} E^{T} R_{2 i} E \Lambda_{i} & -\alpha_{1} \Lambda_{i}^{T} E^{T} R_{2 i} E \Lambda_{i} & 0 & 0 \\
-\alpha_{1} \Lambda_{i}^{T} E^{T} R_{2 i} E \Lambda_{i} & 2 \alpha_{1} \Lambda_{i}^{T} E^{T} R_{2 i} E \Lambda_{i} & -\alpha_{1} \Lambda_{i}^{T} E^{T} R_{2 i} E \Lambda_{i} & 0 \\
0 & -\alpha_{1} \Lambda_{i}^{T} E^{T} R_{2 i} E \Lambda_{i} & \alpha_{1} \Lambda_{i}^{T} E^{T} R_{2 i} E \Lambda_{i}+\alpha_{2} \Lambda_{i}^{T} E^{T} R_{3 i} E \Lambda_{i} & -\alpha_{2} \Lambda_{i}^{T} E^{T} R_{3 i} E \Lambda_{i} \\
0 & 0 & -\alpha_{2} \Lambda_{i}^{T} E^{T} R_{3 i} E \Lambda_{i} & \alpha_{2} \Lambda_{i}^{T} E^{T} R_{3 i} E \Lambda_{i}
\end{array}\right] \\
& =\left[\begin{array}{c}
I \\
-I \\
0 \\
0
\end{array}\right]\left(-\alpha_{1} \Lambda_{i}^{T} E^{T} R_{2 i} E \Lambda_{i}\right)\left[\begin{array}{llll}
I & -I & 0 & 0
\end{array}\right]+\left[\begin{array}{c}
0 \\
I \\
-I \\
0
\end{array}\right]\left(-\alpha_{1} \Lambda_{i}^{T} E^{T} R_{2 i} E \Lambda_{i}\right)\left[\begin{array}{llll}
0 & I & -I & 0
\end{array}\right] \\
& +\left[\begin{array}{c}
0 \\
0 \\
I \\
-I
\end{array}\right]\left(-\alpha_{2} \Lambda_{i}^{T} E^{T} R_{3 i} E \Lambda_{i}\right)\left[\begin{array}{llll}
0 & 0 & I & -I
\end{array}\right] \leq\left[\begin{array}{c}
I \\
-I \\
0 \\
0
\end{array}\right]\left(-\Lambda_{i}^{T} E^{T}-E \Lambda_{i}+\alpha h R_{2 i}^{-1}\right)\left[\begin{array}{llll}
I & -I & 0 & 0
\end{array}\right] \\
& +\left[\begin{array}{c}
0 \\
I \\
-I \\
0
\end{array}\right]\left(-\Lambda_{i}^{T} E^{T}-E \Lambda_{i}+\alpha h R_{2 i}^{-1}\right)\left[\begin{array}{llll}
0 & I & -I & 0
\end{array}\right]+\left[\begin{array}{c}
0 \\
0 \\
I \\
-I
\end{array}\right]\left(-\Lambda_{i}^{T} E^{T}-E \Lambda_{i}+(h-\alpha h) R_{3 i}^{-1}\right)\left[\begin{array}{llll}
0 & 0 & I & -I
\end{array}\right] .
\end{aligned}
$$

Let $\bar{Q}_{1 i j}=\Lambda_{i}^{T} Q_{1 j} \Lambda_{i}, \bar{Q}_{2 i j}=\Lambda_{i}^{T} Q_{2 j} \Lambda_{i}, \bar{Q}_{3 i j}=\Lambda_{i}^{T} Q_{3 i} \Lambda_{i}$, $\bar{R}_{2 i}=R_{2 i}^{-1}, \bar{R}_{3 i}=R_{3 i}^{-1}, \bar{U}_{1 i}=\Lambda_{i}^{T} U_{1} \Lambda_{i}^{T}, \bar{U}_{2 i}=\Lambda_{i}^{T} U_{2} \Lambda_{i}^{T}$, and $\bar{W}=W^{-1}$; then by using Schur complement of Lemma 6 , we have that (69) and (70) hold if (63) and (64) hold. Intuitively, we can verify that (66) and (67) are equivalent to (25) and (26).

On the other hand, it is from (65) that for $\forall j \neq i, \gamma_{i j} \neq 0$,

$$
\frac{W}{N-1}>\gamma_{i j} R_{2 j}
$$

It should also be noted that if $\gamma_{i j}=0,(74)$ always holds. Thus, summing (74) for all $j \in \ell, j \neq i$, implies that

$$
\sum_{j=1, j \neq i}^{N} \gamma_{i j} R_{2 j}-W<0 .
$$

Similarly, we can also obtain

$$
\sum_{j=1, j \neq i}^{N} \gamma_{i j} R_{3 j}-W<0 .
$$


TABLE 1: The maximum allowable upper bound for different $\gamma_{11}$ and time-invariant delay.

\begin{tabular}{lcccc}
\hline Method & $\gamma_{11}$ & -0.8 & -1 & -1.8 \\
\hline$[12]$ & $h_{\max }$ & 0.5562 & 0.5463 & 0.5349 \\
{$[14]$} & $h_{\max }$ & 0.5812 & 0.5760 & 0.5663 \\
Corollary 12 & $h_{\max }$ & 0.7115 & 0.6712 & 0.5886 \\
\hline
\end{tabular}

TABLE 2: The maximum allowable upper bound for different $\gamma_{11}$ and $h_{d}=0.5$.

\begin{tabular}{lccccc}
\hline Method & $\gamma_{11}$ & -0.1 & -0.5 & -0.8 & -1 \\
\hline$[12]$ & $h_{\max }$ & 0.5420 & 0.4897 & 0.4760 & 0.4704 \\
{$[14]$} & $h_{\max }$ & 0.5952 & 0.5757 & 0.5656 & 0.5602 \\
Corollary 11 & $h_{\max }$ & 0.8544 & 0.6535 & 0.6034 & 0.5826 \\
\hline
\end{tabular}

TABLE 3: The maximum allowable upper bound for different $\gamma_{11}$ and $h_{d}=1.5$.

\begin{tabular}{lccccc}
\hline Method & $\gamma_{11}$ & -0.1 & -0.5 & -0.8 & -1 \\
\hline$[12]$ & $h_{\max }$ & 0.3860 & 0.3860 & 0.3860 & 0.3860 \\
{$[14]$} & $h_{\max }$ & 0.5828 & 0.5711 & 0.5631 & 0.5582 \\
Corollary 11 & $h_{\max }$ & 0.7445 & 0.6333 & 0.5943 & 0.5772 \\
\hline
\end{tabular}

Since $\gamma_{i i}<0, R_{2 i}>0$ and $R_{3 i}>0$, it can be verified that (65) guarantees (24). This completes the proof.

To show how to decide the parameter $\alpha$ optimally, we construct the following iterative algorithm for Theorem 9. Following a similar way, we can obtain the iterative algorithms for other theorems and corollaries.

Algorithm 15. Select optimal parameter $\alpha$ for maximum delay h.

Step 1. For a prescribed scalar $h_{d}$, choose appropriate step lengths $\Delta h$ and $\Delta \alpha$ for $h$ and $\alpha$, such that (22)(27) in Theorem 9 hold. Set $k$ as the iterative times, and select the initial values $k=1, h_{0}=\Delta h$, and $\alpha_{0}=$ $\Delta \alpha$. Let the upper bound $h=h_{0}+\Delta h$.

Step 2. Let $\alpha=k \Delta \alpha$; if LMIs (22)-(27) are feasible, go to Step 3; otherwise, go to Step 4.

Step 3. Update $h_{0}, \alpha_{0}, k$ and $h$ as $h_{0}=h, \alpha_{0}=\alpha, k=1$, and $h=h_{0}+\Delta h$, go to Step 2 .

Step 4. Set $k=k+1$. If $k \Delta \alpha<1$; then go to Step 2; otherwise, stop.

From the above algorithm, the output $\alpha_{0}$ is the optimal $\alpha$ to ensure the delay upper bound $h$ to achieve its maximum value $h_{0}$.

\section{Numerical Examples}

In this section, we provide three examples to clarify the applicability and less conservatism of the results proposed in this paper.
TABLE 4: The maximum allowable upper bound for different $\gamma_{11}$ and time-invariant delay.

\begin{tabular}{lccccccc}
\hline Method & $\gamma_{11}$ & -0.6 & -0.55 & -0.5 & -0.45 & -0.4 & -0.35 \\
\hline$[20]$ & $h_{\max }$ & 1.0808 & 1.0842 & 1.0878 & 1.0918 & 1.0960 & 1.1006 \\
{$[17](k=0.5)$} & $h_{\max }$ & 1.1588 & 1.1587 & 1.1587 & 1.1587 & 1.1587 & 1.1587 \\
Corollary 12 & $h_{\max }$ & 1.1769 & 1.1758 & 1.1748 & 1.1737 & 1.1727 & 1.1716 \\
\hline
\end{tabular}

TABLE 5: The maximum allowable upper bound for different $\gamma$ with $h_{d}=0$.

\begin{tabular}{lccccc}
\hline Method & $\gamma$ & 0.5 & 1 & 1.5 & 2 \\
\hline$[12]$ & $h_{\max }$ & 0.4165 & 0.4739 & 0.4926 & 0.5022 \\
Theorem 9 & $h_{\max }$ & 0.4712 & 0.5364 & 0.5581 & 0.5690 \\
\hline
\end{tabular}

Example 1. Consider the nominal Markovian jump systems (55) with two modes and the following parameters:

$$
\begin{array}{ll}
A_{1}=\left[\begin{array}{ll}
-3.4888 & 0.8057 \\
-0.6451 & -3.2684
\end{array}\right], & A_{2}=\left[\begin{array}{cc}
-2.4898 & 0.2895 \\
1.3396 & -0.0211
\end{array}\right], \\
A_{d 1}=\left[\begin{array}{ll}
-0.8620 & -1.2919 \\
-0.6841 & -2.0729
\end{array}\right], & A_{d 2}\left[\begin{array}{cc}
-2.8306 & 0.4978 \\
-0.8436 & -1.0115
\end{array}\right] .
\end{array}
$$

The transition rate matrix is given as

$$
\Gamma=\left[\begin{array}{cc}
\gamma_{11} & \gamma_{12} \\
0.8 & -0.8
\end{array}\right]
$$

It is noted that if $E=I$, descriptor Markovian jump time-delay systems (55) reduce to the standard state-space Markovian jump time-delay systems. For the time-invariant delay, the stochastic stability problem can be solved by Corollary 12. Table 1 shows the compared results on the maximum allowable upper bound for different $\gamma_{11}$ with the recent results $[12,14]$. For $h_{d}=0.5$ and $h_{d}=1.5$, the maximum upper bound on allowable time delay is shown with given $\gamma_{11}$ in Tables 2 and 3, respectively.

As shown in the above tables, the methods in this paper have advantages over some previous ones in that the obtained maximum upper bound for allowable time delay is much larger. Therefore, the developed results have less conservatism than references $[12,14]$.

Example 2. Consider the continuous-time descriptor Markovian jump time-delay systems (59) with two modes and the following parameters:

$$
\begin{gathered}
E=\left[\begin{array}{ll}
1 & 0 \\
0 & 0
\end{array}\right], \quad A_{1}=\left[\begin{array}{cc}
0.4972 & 0 \\
0 & -0.9541
\end{array}\right], \\
A_{2}=\left[\begin{array}{cc}
0.5121 & 0 \\
0 & -0.7215
\end{array}\right], \\
A_{d 1}=\left[\begin{array}{cc}
-1.010 & 1.5415 \\
0 & 0.5449
\end{array}\right], \quad A_{d 2}=\left[\begin{array}{cc}
-0.8521 & 1.9721 \\
0 & 0.4321
\end{array}\right] .
\end{gathered}
$$


The transition rate matrix is given as

$$
\Gamma=\left[\begin{array}{cc}
\gamma_{11} & \gamma_{12} \\
0.3 & -0.3
\end{array}\right] .
$$

Compared with the existing results [17,20], we find that Theorem 14 in this paper reaches the much larger bounds than the aforementioned results. Therefore, we can conclude that Theorem 14 in this paper is less conservative than the results in references $[17,20]$. The compared results can be seen in Table 4.

Example 3. Consider a Markovian jump system (1) with two modes and the following parameters:

$$
\begin{aligned}
A_{1}=\left[\begin{array}{cc}
-2.2460 & -1.4410 \\
-1.5937 & -2.9289
\end{array}\right], & A_{2}=\left[\begin{array}{cc}
-1.8999 & 0.8156 \\
0.6900 & -0.7881
\end{array}\right], \\
A_{d 1}=\left[\begin{array}{cc}
-0.7098 & 1.1908 \\
0.6686 & -3.2025
\end{array}\right], & A_{d 2}=\left[\begin{array}{ll}
-1.5198 & -1.6041 \\
-0.1567 & -1.2427
\end{array}\right], \\
C_{1}=\left[\begin{array}{cc}
-0.3775 & -0.2959
\end{array}\right], & C_{2}=\left[\begin{array}{ll}
-1.4751 & -0.2340
\end{array}\right], \\
C_{d 1}=\left[\begin{array}{ll}
0 & 0
\end{array}\right], & C_{d 2}=\left[\begin{array}{ll}
0 & 0
\end{array}\right], \\
B_{w 1}=\left[\begin{array}{ll}
0.0403 \\
0.6771
\end{array}\right], & B_{w 2}=\left[\begin{array}{c}
0.5689 \\
-0.2556
\end{array}\right], \\
D_{w 1}=0.1184, & D_{w 2}=0.3148
\end{aligned}
$$

The transition matrix is given as

$$
\Gamma=\left[\begin{array}{cc}
-3 & 3 \\
0.6 & -0.6
\end{array}\right] .
$$

When $E=I$, we assume that $B_{1}=B_{2}=0, D_{1}=D_{2}=0$, and $h_{d}=0$; the compared results for different $\gamma>0$ are given in Table 5. Obviously, the BRL obtained in this paper is less conservative than that in [12].

When we take $E=\left[\begin{array}{ll}1 & 0 \\ 0 & 0\end{array}\right]$, then $E_{L}=E_{R}=\left[\begin{array}{ll}1 & 0\end{array}\right]$ satisfies $E=E_{L} E_{R}^{T}$. The coefficient matrices of the input vector can be given as

$$
\begin{array}{cc}
B_{1}=\left[\begin{array}{c}
1.5 \\
0.99
\end{array}\right], & B_{2}=\left[\begin{array}{c}
1.2 \\
0.99
\end{array}\right] \\
D_{1}=0.5, & D_{2}=-0.3 .
\end{array}
$$

In this case, we take $V=\left[\begin{array}{ll}0 & 1\end{array}\right], U=\left[\begin{array}{l}0 \\ 1\end{array}\right], \epsilon_{1}=1.5$, and $\epsilon_{2}=1.8$.

By applying the LMIs conditions in Theorem 14, the minimum attenuation level is $\gamma_{\min }=1.15$ under the assumption $h=0.35$ and $h_{d}=0.3$, and the mode-dependent state feedback controller gain can be obtained. Consider

$$
\begin{aligned}
K_{1} & =\left[\begin{array}{ll}
2.8174 & 0
\end{array}\right], & K_{2} & =\left[\begin{array}{ll}
-63.9993 & -0.0246
\end{array}\right], \\
K_{d 1} & =\left[\begin{array}{ll}
-0.6996 & 0
\end{array}\right], & K_{d 2} & =\left[\begin{array}{ll}
-50.3973 & 0.0141
\end{array}\right] .
\end{aligned}
$$

Therefore, it is easy to see that our results are effective.

\section{Conclusions}

This paper has studied the $H_{\infty}$ control problem for continuous-time descriptor Markovian jump systems with time-varying delay. By constructing novel LyapunovKrasovskii functionals, delay-dependent stability criteria and BRL have been developed under which the underlying systems are regular, impulse-free, and stochastically stable with a prescribed $H_{\infty}$ performance level. Compared with other time-varying delay results based on Jensen inequality, the obtained results in this paper have advantages over some previous results, which is partially attributed to the reciprocally convex combination method. The illustrated examples show that the maximum allowable upper bound of time delay is much larger than some latest results.

\section{Conflict of Interests}

The authors declare that there is no conflict of interests regarding the publication of this paper.

\section{Acknowledgments}

This work was supported by the National Natural Science Foundation of China under Grant no. 61273008 and the Natural Science Foundation of Liaoning Province under Grant no. 201202063, respectively.

\section{References}

[1] K. Gu, V. Kharitonov, and J. Chen, Stability of Time-Delay Systems, Birkhauser, Boston, Mass, USA, 2003.

[2] E. Fridman, "New Lyapunov-Krasovskii functionals for stability of linear retarded and neutral type systems," Systems \& Control Letters, vol. 43, no. 4, pp. 309-319, 2001.

[3] Q.-L. Han, "Robust stability of uncertain delay-differential systems of neutral type," Automatica, vol. 38, no. 4, pp. 719-723, 2002.

[4] Y. He, M. Wu, J.-H. She, and G.-P. Liu, "Delay-dependent robust stability criteria for uncertain neutral systems with mixed delays," Systems \& Control Letters, vol. 51, no. 1, pp. 57-65, 2004.

[5] X.-G. Li, X.-J. Zhu, A. Cela, and A. Reama, "Stability analysis of neutral systems with mixed delays," Automatica, vol. 44, no. 11, pp. 2968-2972, 2008.

[6] X.-L. Zhu and G.-H. Yang, "New results of stability analysis for systems with time-varying delay," International Journal of Robust and Nonlinear Control, vol. 20, no. 5, pp. 596-606, 2010.

[7] H. Shao, "New delay-dependent stability criteria for systems with interval delay," Automatica, vol. 45, no. 3, pp. 744-749, 2009.

[8] P. Park, J. W. Ko, and C. Jeong, "Reciprocally convex approach to stability of systems with time-varying delays," Automatica, vol. 47, no. 1, pp. 235-238, 2011.

[9] E.-K. Boukas, Control of Singular Systems with Random Abrupt Changes, Springer, Berlin, Germany, 2008.

[10] S. Xu and J. Lam, Robust Control and Filtering of Singular Systems, vol. 332 of Lecture Notes in Control and Information Sciences, Springer, Berlin, Germany, 2006.

[11] Z. Fei, H. Gao, and P. Shi, "New results on stabilization of Markovian jump systems with time delay," Automatica, vol. 45, no. 10, pp. 2300-2306, 2009. 
[12] S. Xu, J. Lam, and X. Mao, "Delay-dependent $H_{\infty}$ control and filtering for uncertain Markovian jump systems with timevarying delays," IEEE Transactions on Circuits and Systems I, vol. 54, no. 9, pp. 2070-2077, 2007.

[13] H. Huang, G. Feng, and X. Chen, "Stability and stabilization of Markovian jump systems with time delay via new Lyapunov functionals," IEEE Transactions on Circuits and Systems I, vol. 59, no. 10, pp. 2413-2421, 2012.

[14] J. Yu, W. Sun, and H. Liu, "Improved $H_{\infty}$ analysis of Markovian jumping stochastic systems with time-varying delays," International Journal of Systems Science, vol. 44, no. 3, pp. 521-532, 2013.

[15] L. Dai, Singular Control Systems, vol. 118 of Lecture Notes in Control and Information Sciences, Springer, Berlin, Germany, 1989.

[16] Z.-G. Wu, J. H. Park, H. Su, and J. Chu, "Delay-dependent passivity for singular Markov jump systems with time-delays," Communications in Nonlinear Science and Numerical Simulation, vol. 18, no. 3, pp. 669-681, 2013.

[17] J. Wang, H. Wang, A. Xue, and R. Lu, "Delay-dependent $H_{\infty}$ control for singular Markovian jump systems with time delay," Nonlinear Analysis. Hybrid Systems, vol. 8, pp. 1-12, 2013.

[18] K. Gu, "An integral inequality in the stability problem of time-delay systems," in Proceedings of the 39th IEEE Confernce on Decision and Control, pp. 2805-2810, Sydney, Australia, December 2000.

[19] E. Uezato and M. Ikeda, "Strict LMI conditions for stability, robust stabilization, and $H_{\infty}$ control of descriptor systems," in Proceedings of the The 38th IEEE Conference on Decision and Control (CDC'99), pp. 4092-4097, December 1999.

[20] Z. Wu, H. Su, and J. Chu, "Delay-dependent stabilization of singular Markovian jump systems with state delay," Journal of Control Theory and Applications, vol. 7, no. 3, pp. 231-236, 2009. 


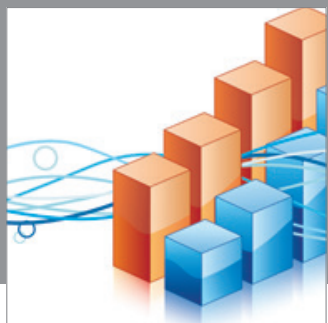

Advances in

Operations Research

mansans

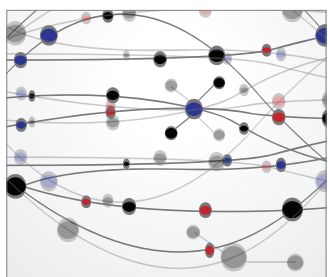

The Scientific World Journal
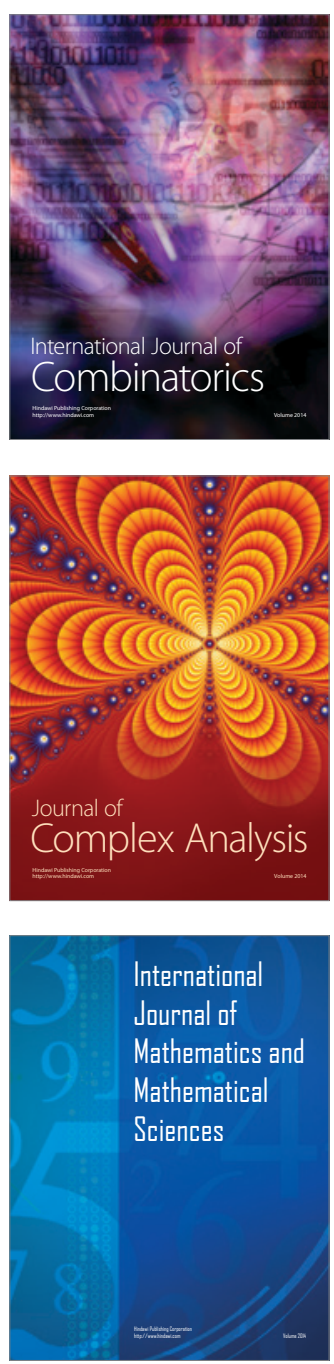
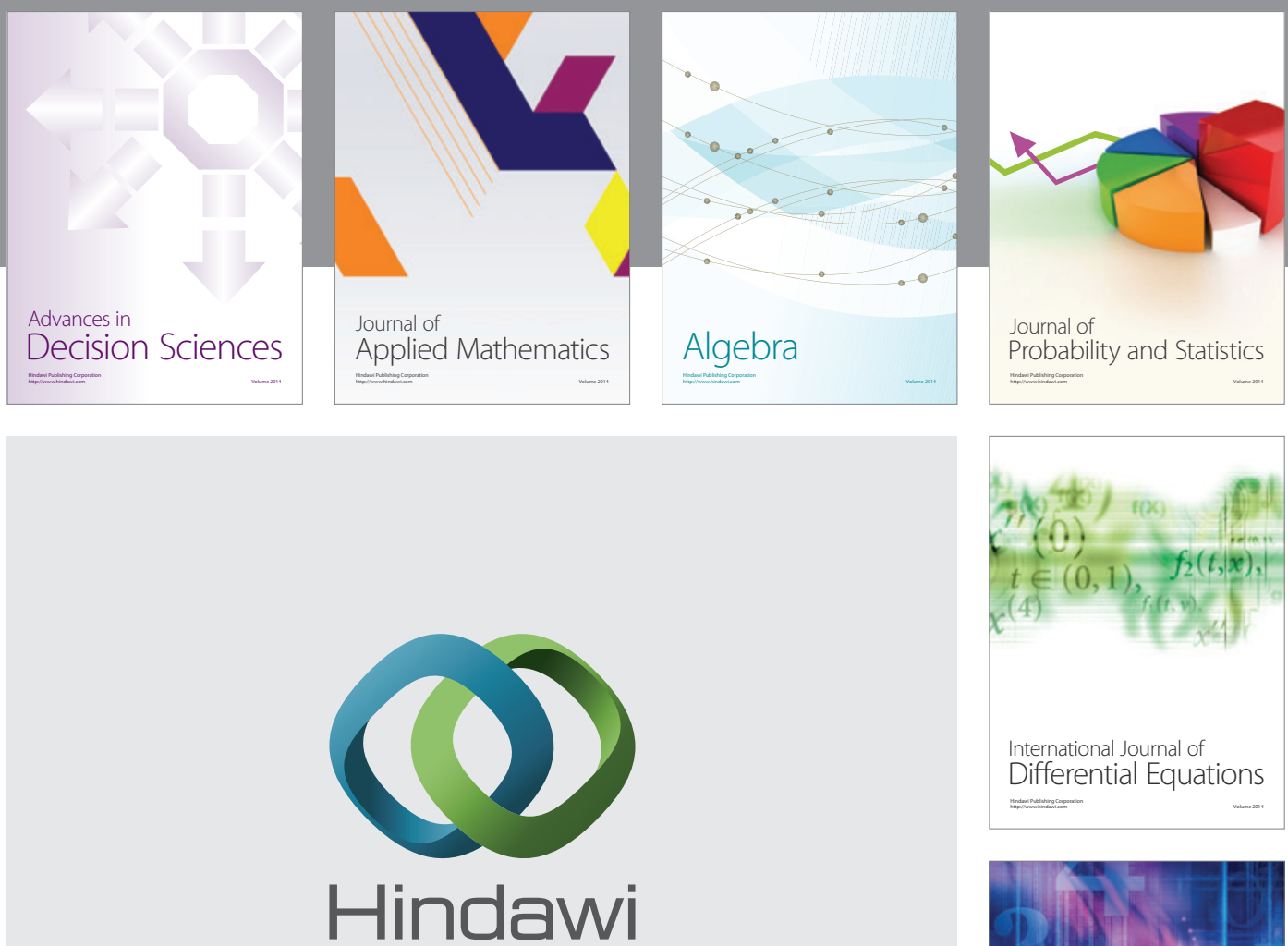

Submit your manuscripts at http://www.hindawi.com
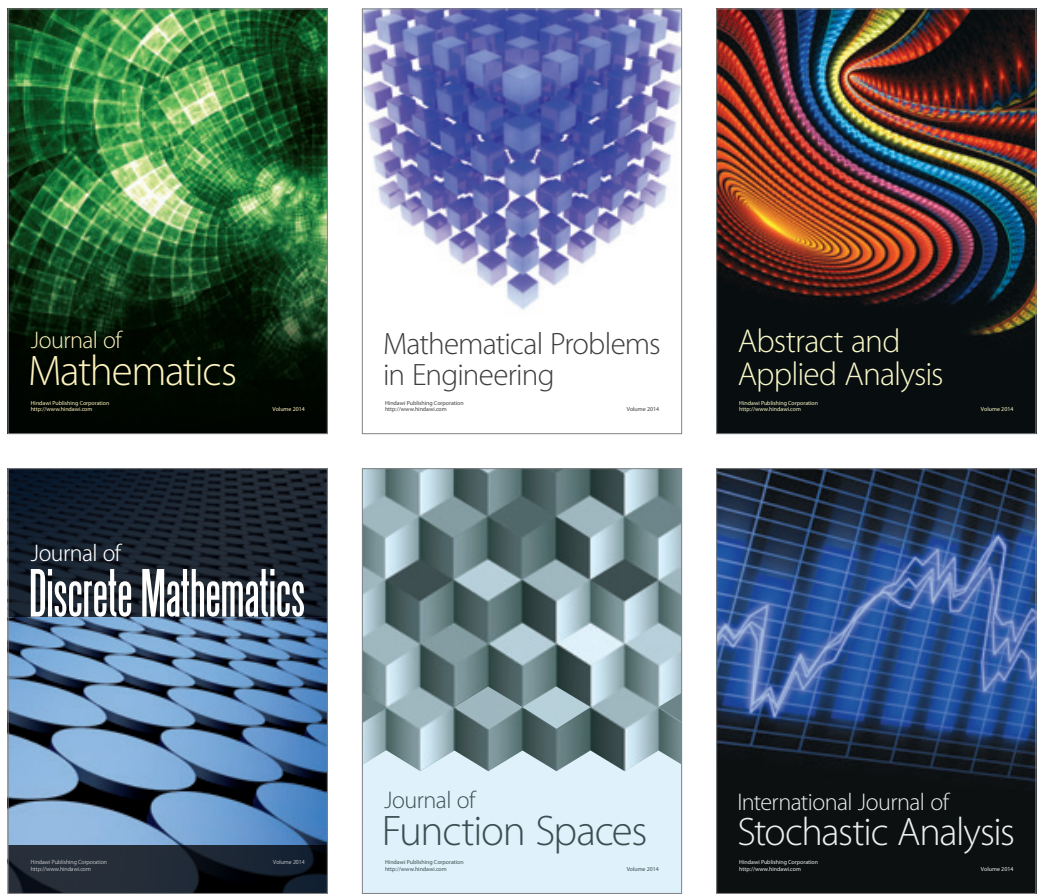

Journal of

Function Spaces

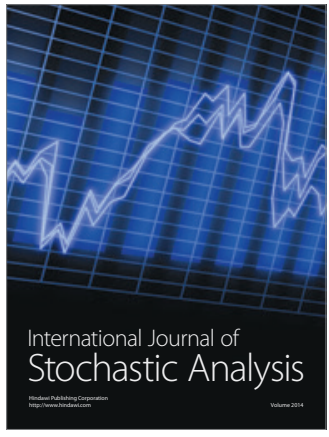

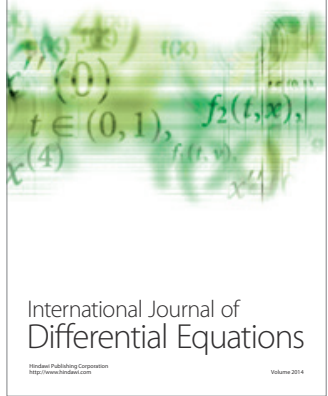
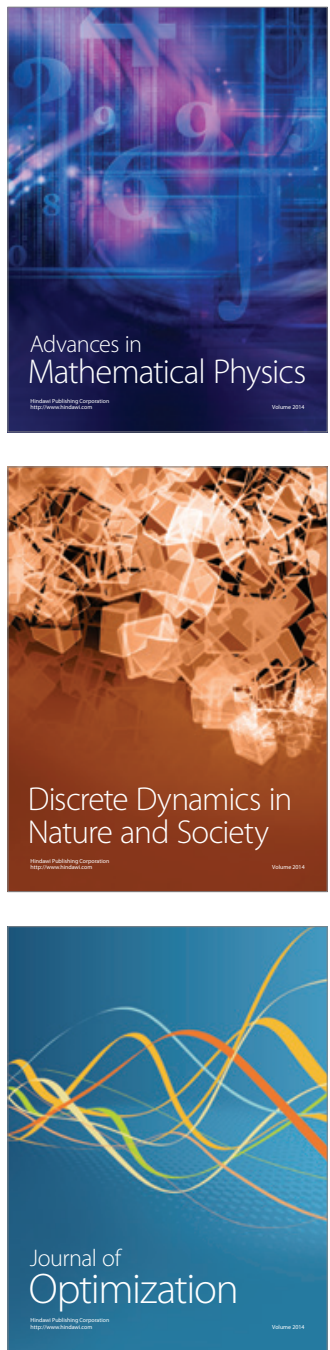